Local Nonlinear Stability of the Steady State in an Isothermal Catalyst

Author(s): Ignacio E. Parra and Jose M. Vega

Source: SIAM Journal on Applied Mathematics, Vol. 48, No. 4 (Aug., 1988), pp. 854-881

Published by: Society for Industrial and Applied Mathematics

Stable URL: http://www.jstor.org/stable/2101551

Accessed: 24/01/2011 04:33

Your use of the JSTOR archive indicates your acceptance of JSTOR's Terms and Conditions of Use, available at http://www.jstor.org/page/info/about/policies/terms.jsp. JSTOR's Terms and Conditions of Use provides, in part, that unless you have obtained prior permission, you may not download an entire issue of a journal or multiple copies of articles, and you may use content in the JSTOR archive only for your personal, non-commercial use.

Please contact the publisher regarding any further use of this work. Publisher contact information may be obtained at http://www.jstor.org/action/showPublisher?publisherCode=siam.

Each copy of any part of a JSTOR transmission must contain the same copyright notice that appears on the screen or printed page of such transmission.

JSTOR is a not-for-profit service that helps scholars, researchers, and students discover, use, and build upon a wide range of content in a trusted digital archive. We use information technology and tools to increase productivity and facilitate new forms of scholarship. For more information about JSTOR, please contact support@jstor.org. 


\title{
LOCAL NONLINEAR STABILITY OF THE STEADY STATE IN AN ISOTHERMAL CATALYST*
}

\author{
IGNACIO E. PARRA AND JOSE M. VEGA
}

\begin{abstract}
A first-order, irreversible, exothermic reaction in a bounded porous catalyst is considered, with smooth boundary, of one, two, or three dimensions. For small Prater and Nusselt numbers, $\beta$ and $\nu$, and a large Sherwood number, $\sigma$, two isothermal models are derived. An analysis of linear stability of the steady states of such models shows that oscillatory instabilities appear for appropriate values of the Damköhler number if the nondimensional activation energy is larger than $\gamma^{*}$ and the Lewis number is sufficiently large, where $\gamma^{*}=4$ if $m=\nu / \beta \sigma \subseteq 1$ and $\gamma^{*}=(m+1)^{2} / m$ if $m>1$. A local Hopf bifurcation analysis is carried out at neutral stability points in order to ascertain whether such bifurcation is subcritical or supercritical.
\end{abstract}

Key words. nonlinear stability, Hopf bifurcation, isothermal catalysts

AMS(MOS) subject classifications. 35B32, 35B35, 80A32

1. Introduction. In this paper we consider a well-known model for the evolution of the reactant concentration $u$ and of the temperature $v$ in a porous catalyst, occupying a region $\Omega$, in which an irreversible, first-order, exothermic reaction is taking place (see Aris [1])

$$
\begin{aligned}
& \frac{\partial u}{\partial t}=\Delta u-\phi^{2} u \exp (\gamma-\gamma / v) \quad \text { in } \Omega, \quad \frac{\partial u}{\partial n}=\sigma(1-u) \quad \text { on } \partial \Omega, \\
& L^{-1} \frac{\partial v}{\partial t}=\Delta v+\beta \phi^{2} u \exp (\gamma-\gamma / v) \text { in } \Omega, \frac{\partial v}{\partial n}=\nu(1-v) \quad \text { on } \partial \Omega .
\end{aligned}
$$

Here, $n$ is the outward unit normal to the smooth boundary of the bounded domain $\Omega \subset \mathbb{R}^{p}$ ( $p=1,2$ or 3 ). The parameters $L$ (Lewis number, the ratio of thermal to material diffusivity), $\phi^{2}$ (Damköhler number, a measure of the reaction rate relative to the diffusion rate), $\gamma$ (activation energy), $\beta$ (chemical heat release or Prater number, $\beta L$ is the ratio of the heat of reaction to the thermal energy of the catalyst), $\sigma$ and $\nu$ (Sherwood and Nusselt numbers, the ratios of the rates of mass and heat transfer between the surface of the catalyst and the external unreacted fluid to the corresponding rates of mass and heat transfer within the catalyst) are positive constants. For nondimensionalization, length is referred to a characteristic dimension of the catalyst, and time to the diffusion time within the catalyst, while concentration and temperature are referred to their respective values in the external unreacted fluid.

We shall consider the limit

$$
L \rightarrow \infty, \quad \gamma \beta \rightarrow 0, \quad \nu \rightarrow 0,
$$

which accounts for the fact that the thermal diffusitivity of the solid catalyst is usually very high and leads to the so-called isothermal models in which the temperature does not depend on the spatial variables; this limit is quite realistic, as has been pointed out in the literature (Aris [1] and references given therein). In addition, in $\$ \$ 2-5$ we shall assume that $\sigma$ is a large parameter, as is frequently the case in practice because the exchange of mass with the external fluid is much faster than through the pores of

\footnotetext{
* Received by the editors August 11, 1986; accepted for publication (in revised form) May 5, 1987. This research was partially supported by the Spanish Comisión Asesora de lnvestigación Científica y Técnica, under grant N/r 2291-83.

$\div$ Escuela Técnica Superior de Ingenieros Aeronáuticos, Universidad Politécnica de Madrid, 28040 Madrid, Spain.
} 
the catalyst; nevertheless, if the size of the catalyst is sufficiently small, $\sigma$ may be of the order of unity, as will be assumed in $\$ 6$. The results below will be valid for arbitrary values of $\gamma$ (which is frequently fairly large) and $\phi^{2}$ (which varies over a wide range in practice).

In $\$ 2$ we shall derive two models which are appropriate for the study of nonlinear stability of the steady states of (1.1), (1.2) under small perturbations. If $\phi^{2}$ is not too large, we shall obtain the following model, which will be referred to as Model 1 in the sequel

$$
\begin{aligned}
& \frac{\partial u}{\partial t}=\Delta u-\phi^{2} u \exp (\gamma-\gamma / v) \quad \text { in } \Omega, \quad u=1 \quad \text { on } \partial \Omega \\
& \frac{d v}{d t}=\lambda \mu(1-v)+\lambda \phi^{2} \exp (\gamma-\gamma / v) \int_{\Omega} u d x .
\end{aligned}
$$

Here, the parameters $\lambda$ and $\mu$ are

$$
\lambda=\beta L / V_{\Omega}, \quad \mu=\nu S_{\Omega} / \beta,
$$

where $V_{\Omega}$ and $S_{\Omega}$ are the volume and the external area of the domain $\Omega\left(S_{\Omega}=V_{\Omega}=2\right.$ if $\Omega=]-1,1[\subset \mathbb{R}$ ).

If $\phi^{2}$ is sufficiently large, then the concentration vanishes to leading order outside a thin boundary layer which is close to $\partial \Omega$, and the following model (Model 2) will be obtained

$$
\begin{aligned}
& \frac{\partial U}{\partial \tau}=\frac{\partial^{2} U}{\partial \xi^{2}}-\Phi^{2} U \exp (\gamma-\gamma / v) \quad \text { in }-\infty<\xi<0, \\
& U=0 \quad \text { at } \xi=-\infty, \quad \frac{\partial U}{\partial \xi}=1-U \text { at } \xi=0, \\
& \frac{d v}{d \tau}=\operatorname{lm}(1-v)+l \Phi^{2} \exp (\gamma-\gamma / v) \int_{-\infty}^{0} U d \xi
\end{aligned}
$$

with

$$
\Phi=\frac{\phi}{\sigma}, \quad l=\frac{\beta L S_{\Omega}}{\sigma V_{\Omega}}, \quad m=\frac{\nu}{\beta \sigma}, \quad \tau=\sigma^{2} t, \quad \xi=\sigma \eta .
$$

Here, $\eta$ is a coordinate along the outward unit normal to $\partial \Omega$ and $U$ is the mean value of the concentration $u$ at time $\tau$ over the surface $\xi=$ constant, which is parallel to $\partial \Omega$ and close to it ( $U=u$ if $\Omega=]-1,1[\subset \mathbb{R}$ ). Observe that Model 2 is independent of the shape of the domain $\Omega$; it depends only on the volume and the external area of $\Omega$, through the parameters $l$ and $m$.

Models 1 and 2 may also be obtained in the limit $\sigma \rightarrow \infty$ from the isothermal model posed by (1.1), (1.4), which is readily obtained from (1.1), (1.2) whenever the temperature $v$ may be considered to be spatially uniform (as is the case, after a short time, under the assumptions that lead to Models 1 and 2, as will be seen in $\S 2$ ); then, (1.4) is obtained from (1.2) upon integration over $\Omega$, application of Green's identity, and substitution of the boundary condition at $\partial \Omega$.

Model 1 was considered, for the slab geometry $(\Omega=]-1,1[\subset \mathbb{R})$, by Amundson and Raymond [2]. In their work, it was apparently proved that the possible instabilities of the steady state are of the nonoscillatory type and that they appear only at those points of the parameter space where the number of steady states changes. A gap in their use of Rouche's Theorem prevents their conclusion from being true. In fact, as we shall see in $\$ 3.1$, oscillatory instabilities do take place in a region of the parameter space. 
Linear stability properties of Model 1 for arbitrary shapes of the domain $\Omega$ in two and three dimensions and of Model 2 will be considered in $\$ \$ 3.2$ and 4 , where oscillatory instabilities will be found again. Local Hopf bifurcation for Model 2 will be considered in $\$ S$. Finally, in $\$ 6$ we shall consider the limit $\sigma=O(1)$.

The results below also apply to some generalizations of the isothermal model (1.1), (1.4), such as that considered by Nieisen and Villadsen [3]. Global stability results for (1.1), (1.4) and: (a) more general kinetic laws and (b) arbitrary positive values of the Sherwood number (not necessarily large) will be considered elsewhere [4]. Finally, let us point out that in the (not quite realistic) limit $\sigma \rightarrow 0, \beta \rightarrow \infty$, $\phi^{2} \beta=O(1)$, concentration (and not temperature) is lumped, and a model (Model 2 of [2]) is obtained, which is essentially the converse of the isothermal model. This model exhibits relaxation oscillations, as was shown by Hastings [5]. Unfortunately, the analysis of relaxation oscillations for the isothermal model (1.1), (1.4) seems to be much more involved than that in [5] (except in the limit $\phi / \sigma \rightarrow \infty$ that will be considered in $\$ 6$, in which the global dynamics of (1.1), (1.4) is essentially twodimensional); such analysis could be of interest in explaining some numerical results in the literature (see [6] and references given therein), since the isothermal model is more realistic than the model considered in [S].

2. Asymptotic derivation of Models 1 and 2. In this section, we shall consider the limit

$$
L \rightarrow \infty, \quad \gamma \beta \rightarrow 0, \quad \nu \rightarrow 0, \quad \sigma \rightarrow \infty
$$

for the problem (1.1), (1.2), with initial conditions

$$
u(x, 0)=\bar{u}(x), \quad v(x, 0)=\tilde{v}(x) \quad \text { in } \Omega,
$$

where the smooth functions $u$ and $v$ satisfy the boundary conditions at $\partial \Omega$.

For the sake of brevity, only the three-dimensional case $\left(\Omega \subset \mathbb{R}^{3}\right)$ will be considered; the results below are also valid in one and two dimensions. Also, we shall consider only the case $\gamma=O(1)$. If $\gamma \gg 1$ and if the initial conditions (2.1) are such that $\gamma\left(\tilde{v}-v_{s}\right)=O(1)\left(v_{s}\right.$ is any steady state temperature of $\left.(1.1),(1.2)\right)$, then the problem (1.1), (1.2) also leads to submodels of Models 1 or 2, depending on whether the following condition is satisfied or not

$$
\phi^{2} \exp \left(\gamma-\gamma / v_{s}\right) \ll \sigma^{2},
$$

as may be seen by means of an analysis which is similar to that given below.

A part of the analysis follows Cohen and Poore [7] and Murray [8].

2.1. Derivation of Model 1. In the distinguished limit $\sigma \sim L \sim \nu^{-1}-\beta^{-1} \gg 1, \gamma \sim 1$, $\phi^{2} \sim 1$, the parameters $\lambda$ and $\mu$, defined in (1.5), are of the order of unity. Let us assume that the initial conditions (2.1) and their first- and second-order derivatives are of the order of unity in $\Omega$. We shall seek the expansions

$$
u=u_{0}+\beta u_{1}+\cdots, \quad v=v_{0}+\beta v_{1}+\cdots,
$$

and distinguish two time scales. For $t \sim \beta, u_{0}$ and $v_{0}$ are given by

$$
\frac{\partial u_{0}}{\partial T}=0, \quad \frac{\partial v_{0}}{\partial T}=\Delta v_{0} \quad \text { in } \Omega, \quad \frac{\partial v_{0}}{\partial n}=0 \quad \text { on } \partial \Omega,
$$

in terms of the time variable $T=\lambda V_{\Omega} t / \beta$. Therefore, $u_{0}=\tilde{u}(x)$ remains constant in this time scale and

$$
v_{0} \rightarrow V_{\Omega}^{-1} \int_{\Omega} \tilde{v}(x) d x \text { as } T \rightarrow \infty
$$


For $t \sim 1, u_{0}$ is given by (1.3) when the subscript 0 is dropped from $u$ and $v$, while $v_{0}$ and $v_{1}$ are given by

$$
\begin{aligned}
& \Delta \psi_{0}=0 \quad \text { in } \Omega, \quad \frac{\partial v_{0}}{\partial n}=0 \text { on } \partial \Omega, \\
& \left(\lambda V_{\Omega}\right)^{-1} \frac{\partial v_{0}}{\partial t}=\Delta v_{1}+\phi^{2} u_{0} \exp \left(\gamma-\gamma / v_{0}\right) \text { in } \Omega, \\
& S_{\Omega} \frac{\partial v_{1}}{\partial n}=\mu\left(1-v_{0}\right) \quad \text { on } \partial \Omega,
\end{aligned}
$$

Equation (2.2) yields $v_{0}=v_{0}(t)$. Then, integration of (2.3) over $\Omega$, application of Green's identity, and substitution of the boundary condition (2.4) lead to (1.4) when the subscript 0 is dropped. Observe that the appropriate initial conditions for Model 1 are

$$
u(0, x)=\tilde{u}(x), \quad v(0)=V_{\Omega}^{-1} \int_{\Omega} \tilde{v}(x) d x,
$$

as comes from matching conditions with the earlier time stage.

If the first-order type of kinetic law is replaced by a general one, $\phi^{2} f(u, v)$, then the analysis above stands after trivial changes.

2.2. Derivation of Model 2. In the distinguished limit $\phi \sim L^{1 / 3} \sim \nu^{-1} \sim \beta^{-1 / 2} \sim \sigma \gg$ $1, \gamma \sim 1$, the parameters $\Phi, l$, and $m$ defined in (1.9) are of the order of unity. In this limit, it will appear a thin layer, of thickness $\sigma^{-1}$, beside the boundary $\partial \Omega$. The solution in this boundary layer will be described in terms of the space variables $\xi, \eta^{1}$, and $\eta^{2}$, where $\xi=\sigma \eta$ and where $\left(\eta, \eta^{1}, \eta^{2}\right)$ is an orthogonal curvilinear coordinate system, defined in a neighborhood $B$ of each point of $\partial \Omega$, which is such that $\eta$ is a coordinate along the outward unit normal to $\partial \Omega$, and $\partial \Omega$ is the parametric surface $\eta=0$. Then, the Laplacian operator is

$$
\Delta=\frac{\partial^{2}}{\partial \eta^{2}}+h^{i j} \frac{\partial^{2}}{\partial \eta^{i} \partial \eta^{j}}+p^{k} \frac{\partial}{\partial \eta^{k}},
$$

where $h^{i j}$ and $p^{k}\left(i, j, k=1\right.$ and 2) are appropriate smooth functions of $\eta, \eta^{1}$, and $\eta^{2}$, defined in the neighborhood $B$.

Let us assume that the initial conditions (2.1) are such that: (a) $\sigma^{2} \tilde{u}(x), \tilde{v}(x)$ and their first- and second-order derivatives are of the order of unity in the outer zone (i.e., outside the boundary layer), and (b) $\tilde{u}\left(\xi, \eta^{1}, \eta^{2}\right), \tilde{v}\left(\xi, \eta^{1}, \eta^{2}\right)$ and their first- and second-order derivatives are of the order of unity in the boundary layer. Then, we shall seek expansions of the form

$$
u=\sigma^{-2} u_{2}+\cdots, \quad v=v_{0}+\sigma^{-1} v_{1}+\cdots
$$

in the outer zone, and of the form

$$
u=\tilde{u}_{0}+\sigma^{-1} \bar{u}_{1}+\cdots, \quad v=\bar{v}_{0}+\sigma^{-1} \bar{v}_{1}+\cdots
$$

in the boundary layer.

Three time scales must be considered. For $t \sim \sigma^{-5}, \bar{u}_{0}, u_{2}$, and $v_{0}$ are seen to remain constant, and $\bar{v}_{0}$ and $\bar{v}_{1}$ are given by

$$
\begin{aligned}
& \frac{\partial \bar{v}_{i}}{\partial T_{1}}=\frac{\partial^{2} \bar{v}_{i}}{\partial \xi^{2}} \text { in }-\infty<\xi<0, \\
& \bar{v}_{i} \rightarrow A_{i}\left(\eta^{1}, \eta^{2}\right) \quad \text { as } \xi \rightarrow-\infty, \quad \frac{\partial \bar{v}_{i}}{\partial \xi}=0 \quad \text { at } \xi=0,
\end{aligned}
$$


For $t \sim 1, u_{0}$ is given by (1.3) when the subscript 0 is dropped from $u$ and $v$, while $v_{0}$ and $v_{1}$ are given by

$$
\begin{aligned}
& \Delta v_{0}=0 \quad \text { in } \Omega, \quad \frac{\partial v_{0}}{\partial n}=0 \quad \text { on } \partial \Omega, \\
& \left(\lambda V_{\Omega}\right)^{-1} \frac{\partial v_{0}}{\partial t}=\Delta v_{1}+\phi^{2} u_{0} \exp \left(\gamma-\gamma / v_{0}\right) \text { in } \Omega, \\
& S_{\Omega \Omega} \frac{\partial v_{1}}{\partial n}=\mu\left(1-v_{0}\right) \quad \text { on } \partial \Omega,
\end{aligned}
$$

Equation (2.2) yields $v_{0}=v_{0}(t)$. Then, integration of (2.3) over $\Omega$, application of Green's identity, and substitution of the boundary condition (2.4) lead to (1.4) when the subscript 0 is dropped. Observe that the appropriate initial conditions for Model 1 are

$$
u(0, x)=\tilde{u}(x), \quad v(0)=V_{\Omega}^{-1} \int_{\Omega} \tilde{v}(x) d x,
$$

as comes from matching conditions with the earlier time stage.

If the first-order type of kinetic law is replaced by a general one, $\phi^{2} f(u, v)$, then the analysis above stands after trivial changes.

2.2. Derivation of Model 2. In the distinguished limit $\phi \sim L^{1 / 3} \sim \nu^{-1} \sim \beta^{-1 / 2} \sim \sigma \gg$ 1, $\gamma \sim 1$, the parameters $\Phi, l$, and $m$ defined in (1.9) are of the order of unity. In this limit, it will appear a thin layer, of thickness $\sigma^{-1}$, beside the boundary $\partial \Omega$. The solution in this boundary layer will be described in terms of the space variables $\xi, \eta^{1}$, and $\eta^{2}$, where $\xi=\sigma \eta$ and where $\left(\eta, \eta^{1}, \eta^{2}\right)$ is an orthogonal curvilinear coordinate system, defined in a neighborhood $B$ of each point of $\partial \Omega$, which is such that $\eta$ is a coordinate along the outward unit normal to $\partial \Omega$, and $\partial \Omega$ is the parametric surface $\eta=0$. Then, the Laplacian operator is

$$
\Delta=\frac{\partial^{2}}{\partial \eta^{2}}+h^{i j} \frac{\partial^{2}}{\partial \eta^{i} \partial \eta^{j}}+p^{k} \frac{\partial}{\partial \eta^{k}},
$$

where $h^{i j}$ and $p^{k}(i, j, k=1$ and 2$)$ are appropriate smooth functions of $\eta, \eta^{1}$, and $\eta^{2}$, defined in the neighborhood $B$.

Let us assume that the initial conditions (2.1) are such that: (a) $\sigma^{2} \tilde{u}(x), \tilde{v}(x)$ and their first- and second-order derivatives are of the order of unity in the outer zone (i.e., outside the boundary layer), and (b) $\tilde{u}\left(\xi, \eta^{1}, \eta^{2}\right), \tilde{v}\left(\xi, \eta^{1}, \eta^{2}\right)$ and their first- and second-order derivatives are of the order of unity in the boundary layer. Then, we shall seek expansions of the form

$$
u=\sigma^{-2} u_{2}+\cdots, \quad v=v_{0}+\sigma^{-1} v_{1}+\cdots
$$

in the outer zone, and of the form

$$
u=\bar{u}_{0}+\sigma^{-1} \bar{u}_{1}+\cdots, \quad v=\bar{v}_{0}+\sigma^{-1} \bar{v}_{1}+\cdots
$$

in the boundary layer.

Three time scales must be considered. For $t \sim \sigma^{-5}, \bar{u}_{0}, u_{2}$, and $v_{0}$ are seen to remain constant, and $\bar{v}_{0}$ and $\bar{v}_{1}$ are given by

$$
\begin{aligned}
& \frac{\partial \bar{v}_{i}}{\partial T_{1}}=\frac{\partial^{2} \bar{v}_{i}}{\partial \xi^{2}} \text { in }-\infty<\xi<0, \\
& \bar{v}_{i} \rightarrow A_{i}\left(\eta^{1}, \eta^{2}\right) \quad \text { as } \xi \rightarrow-\infty, \quad \frac{\partial \bar{v}_{i}}{\partial \xi}=0 \quad \text { at } \xi=0,
\end{aligned}
$$


Then, integration of (2.10) over $\Omega$, application of Green's identity, and substitution of the boundary condition (2.13) lead to (1.8) if the subscript 0 is dropped and if

$$
U=S_{\Omega}^{-1} \int_{S(\xi)} \bar{u}_{0}\left(\xi, \eta^{\prime}, \eta^{2}\right) d A(\xi),
$$

where $S(\xi)$ is the parametric surface $\xi=$ constant; to obtain (1.8) it is necessary to take into account that if $\sigma$ is large in comparison with the maximum normal curvature of $\partial \Omega$, then the parametric surfaces $\xi=$ constant coincide with the surface $\partial \Omega$ in first approximation and, to leading order,

$$
\int_{-\infty}^{0} U d \xi=\int_{-\infty}^{0}\left(\int_{S(\xi)} \bar{u}_{0} d A(\xi)\right) d \xi=\int_{a \mathfrak{N}}\left(\int_{-\infty}^{0} \bar{u}_{0} d \xi\right) d A .
$$

Finally, $U$ satisfies (1.6) and (1.7), as is seen when (2.7) and the boundary conditions (2.8) are integrated over $S(\xi)$. The appropriate initial conditions for Model 2 are

$$
U(0, \xi)=S_{\Omega}^{-1} \int_{S(\xi)} \tilde{u}\left(\xi, \eta^{\prime}, \eta^{2}\right) d A(\xi), \quad v(0)=V_{\Omega}^{-1} \int_{\Omega} \tilde{v}(x) d x,
$$

as they are obtained from matching conditions with earlier time stages.

Observe that the linearity in $u$ of the reaction term is essential in the analysis above. For general kinetic laws, of the type $\phi^{2} f(u, v),(1.8)$ must be replaced by

$$
\frac{d v}{d \tau}=m l(1-v)+l \Phi^{2} S_{\Omega}^{-1} \int_{\partial \Omega}\left[\int_{-\infty}^{o} f(U, v) d \xi\right] d A,
$$

where $U=\bar{u}_{0}$, because the dependence of $\bar{u}_{0}$ on $\eta^{1}$ and $\eta^{2}$ does not disappear in this time scale. Nevertheless, even for general kinetic laws, $v_{0}$ is lumped after a short initial stage (see (2.6)); therefore, the isothermal model (1.1), (1.4) applies also in this case, as was seen in the Introduction.

Finally, observe that we imposed some constraints on initial conditions to obtain Model 2, which imply that initial conditions are not too far from the steady state under consideration. If $\gamma=O(1)$, a more involved asymptotic analysis would show that Model 2 is obtained, from the nonisothermal model, for arbitrary initial conditions. Unfortunately, this is not necessarily true if $\gamma \gg 1$, as is frequently the case in practice.

\section{Linear stability for Model 1.}

3.1. The slab geometry. Let us consider Model 1 in $\Omega=]-1,1[\subset R$. It may be seen that no properties concerning the asymptotic behavior (as $t \rightarrow \infty$ ) are lost if we consider the symmetric case, i.e.,

$$
\begin{aligned}
& \frac{\partial u}{\partial t}=\frac{\partial^{2} u}{\partial x^{2}}-\phi^{2} u \exp (\gamma-y / v) \quad \text { in } 0<x<1, \\
& \frac{\partial u}{\partial x}=0 \quad \text { at } x=0, \quad u=1 \quad \text { at } x=1, \\
& \frac{d v}{d t}=\lambda \mu(1-v)+2 \lambda \phi^{2} \exp (\gamma-\gamma / v) \int_{0}^{1} u d x
\end{aligned}
$$

The steady state solutions are

$$
u_{s}=\cosh \left(\phi_{s} x\right) / \cosh \phi_{s}, \quad v_{s}=1+2 \mu^{-1} \phi_{s} \tanh \phi_{s},
$$

in terms of the parameter $\phi_{s}$, which is given by

$$
\phi^{2}=\phi_{s}^{2} \exp \left(\gamma / v_{s}-1\right) .
$$


The linearized problem around the steady state has nontrivial solutions of the form $u-u_{\mathrm{s}}=X(x) \exp (\omega t), v-v_{s}=\gamma \exp (\omega t)$, if and only if $\omega$ satisfies

$$
\omega(\omega+\lambda \mu)=\frac{2 \lambda \mu^{2} \gamma \phi_{s}}{\left(\mu+2 \phi_{s} \tanh \phi_{s}\right)^{2}}\left(\frac{\phi_{s}^{3} \tanh \sqrt{\omega+\phi_{s}^{2}}}{\sqrt{\omega+\phi_{s}^{2}}}+\left(\omega-\phi_{s}^{2}\right) \tanh \phi_{s}\right),
$$

$$
\gamma=2\left(\mu \cosh \phi_{s}+2 \phi_{s} \sinh \phi_{s}\right)^{2} / \mu \phi_{s}\left(2 \phi_{s}+\sinh 2 \phi_{s}\right),
$$

for $\omega \neq 0$ and for $\omega=0$, respectively. For fixed values of $\gamma, \lambda$, and $\mu$, the neutral stability points of the response curve, $v_{s}-\phi$, are (if they exist) those corresponding to values of $\phi_{s}$ which are: (i) solutions of (3.2), or (ii) solutions of (3.1) for a purely imaginary value of $\omega, \omega=i \Omega$. Neutral stability points are sketched in Fig. 1 for fixed values of $\lambda$ and $\mu$.

The upper curve in the sketches of Fig. 1 is given by (3.2), and it does not depend on $\lambda$. The function (3.2) has a unique minimum, $\gamma_{0}$, which is reached at $\phi_{\mathrm{s}}=\phi_{\mathrm{s} 0}$, where $\phi_{s o}$ is the unique solution of

$$
\mu=2 \phi_{s} \frac{\cosh \phi_{s}\left(\sinh ^{2} \phi_{s}+2 \phi_{s}^{2}\right)+\phi_{s} \sinh \phi_{s}}{\sinh \phi_{s}\left(\cosh ^{2} \phi_{s}-2 \phi_{s}^{2}\right)+3 \phi_{s} \cosh \phi_{s}} .
$$

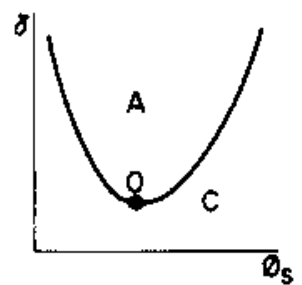

(a)

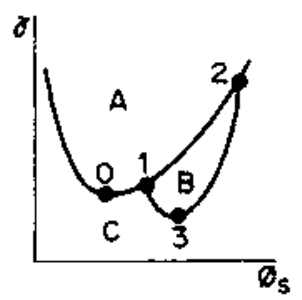

(d)

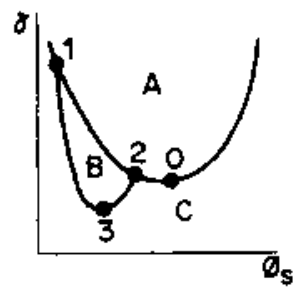

(g)

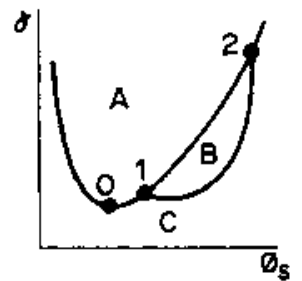

(b)

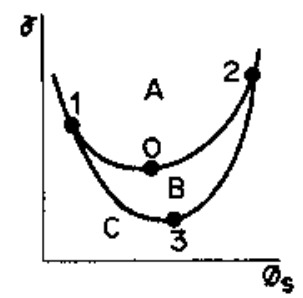

(e)

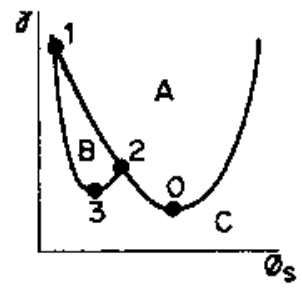

(h)

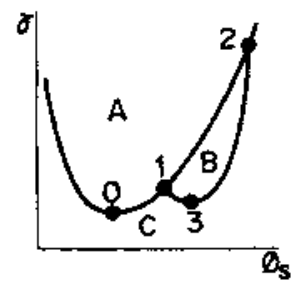

(c)

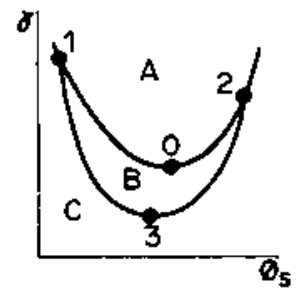

(f)

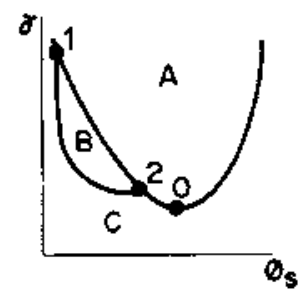

(i)

FIG. 1. Curves of neutral stability points of Model 1 (sjab geometry) for fixed values of $\lambda$ and $\mu$. (a) $\mu$ arbitrary and $\lambda \mu<(\lambda \mu)_{n 1}$; (b) $\mu<\mu_{c}$ and $(\lambda \mu)_{m 1}<\lambda \mu<(\lambda \mu)_{13}$; (c) $\mu<\mu_{r}$ and $(\lambda \mu)_{13}<\lambda \mu<(\lambda \mu)_{03}$; (d) $\mu<\mu_{1}$ and $(\lambda \mu)_{03}<\lambda \mu<(\lambda \mu)_{0 \mathrm{r}}$; (e) $\mu<\mu_{\text {c }}$ and $(\lambda \mu)_{01}<\lambda \mu<(\lambda \mu)_{12}$; (f) $\mu<\mu_{\text {c }}$ and $(\lambda \mu)_{12}<\lambda \mu$, $\mu=\mu_{c}$ and $(\lambda \mu)_{m}<\lambda \mu$, or $\mu>\mu_{c}$ and $\{\lambda \mu)_{02}<\lambda \mu ;(\mathrm{g}) \mu>\mu_{c}$ and $(\lambda \mu)_{03}<\lambda \mu<\left(\lambda_{\mu}\right)_{02} ;$ (h) $\mu>\mu_{c}$ and $(\lambda \mu)_{23}<\lambda \mu<(\lambda \mu)_{03} ;(\mathrm{i}) \mu>\mu_{c}$ and $(\lambda \mu)_{m}<\lambda \mu<(\lambda \mu)_{23}$. 
The following equations are obtained from the real and imaginary parts of the equation that results when (3.1) is multiplied by $\omega+\phi_{\text {; }}^{2}$ and $\omega$ is replaced by i $\Omega$

$$
\begin{gathered}
\gamma=\frac{\Omega\left(\lambda \mu \phi_{s}^{2}-\Omega^{2}\right)\left(\mu+2 \phi_{s} \tanh \phi_{s}\right)^{2}(\cosh a+\cos b)}{\lambda \mu^{2} \phi_{s}^{4}(b \sinh a+a \sin b)}, \\
\frac{\lambda \mu+\phi_{s}^{2}}{\Omega^{2}-\lambda \mu \phi_{s}^{2}}=\frac{8 \phi_{s}^{3}(a \sinh a-b \sin b)-\left(a^{2}+b^{2}\right)^{2}(\cosh a+\cos b) \tanh \phi_{s}}{8 \Omega \phi_{s}^{3}(b \sinh a+a \sin b)},
\end{gathered}
$$

where $a>0$ and $b \geqq 0$ are given by

$$
a^{2}=2\left(\phi_{3}^{2}+\sqrt{\Omega^{2}+\phi_{s}^{4}}\right), \quad b^{2}=2\left(-\phi_{s}^{2}+\sqrt{\Omega^{2}+\phi_{s}^{4}}\right) .
$$

For $\Omega=0$, (3.3) leads to (3.2), and (3.4) yields

$$
\lambda \mu=4 \phi_{s}^{2}\left(2 \phi_{s}+\sinh 2 \phi_{s}\right) /\left(3 \sinh 2 \phi_{s}-6 \phi_{s}-4 \phi_{s}^{2} \tanh \phi_{s}\right) .
$$

If $\lambda \mu<(\lambda \mu)_{m}=8.889 \cdots$, (3.5) has no positive solution and neither has (3.4). If $\lambda \mu>(\lambda \mu)_{m}$, (3.5) has two positive solutions, $\phi_{s,}$ and $\phi_{s 2}$; for $\phi_{s 1}<\phi_{s}<\phi_{s 2},(3.4)$ is seen to define a nonnegative function

$$
\Omega=\Omega\left(\lambda \mu, \phi_{s}\right)
$$

which is such that $\Omega\left(\lambda \mu, \phi_{s 1}\right)=\Omega\left(\lambda \mu, \phi_{s 2}\right)=0, \Omega\left(\lambda \mu, \phi_{s}\right)>0$ for $\phi_{11}<\phi_{s}<\phi_{s 2}$.

The lower curve in the sketches of Fig. 1, which does not exist if $\lambda \mu<(\lambda \mu)_{m}$, is given by (3.3) with $\Omega$ as given by (3.6). The coordinates of points 1 and 2 are $\left(\phi_{, 1}, \gamma_{1}\right)$ and $\left(\phi_{s 2}, \gamma_{2}\right)$, where $\gamma_{1}$ and $\gamma_{2}$ are given by (3.2) with $\phi_{s}=\phi_{11}$ and $\phi_{s}=\phi_{s 2}$, respectively. Point 3 (when it exists) is the unique minimum of the function $\phi_{3} \rightarrow \gamma\left(\phi_{4}, \lambda_{\mu}, \mu\right)$ defined by (3.3) and (3.6); its coordinates must be computed numerically.

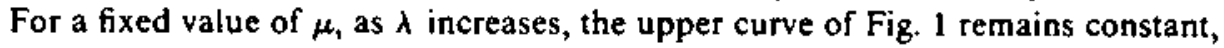
$\phi_{s 1}$ decreases, $\phi_{s 2}$ increases, and any point of the lower curve moves down. The functions $\gamma_{i}=\gamma_{i}(\lambda \mu, \mu)$, for $i=0,1,2$, and 3, are as plotted in Fig. 2, where $\gamma_{1} / \lambda_{\mu} \rightarrow$ $\mu / 15, \gamma_{2} / \sqrt{\lambda \mu} \rightarrow 2 \sqrt{3} / \mu$ and $\gamma_{3} \rightarrow 4$, as $\lambda \rightarrow \infty$. The shape of the neutral stability curves, $\gamma-\phi_{s}$ is as one of the sketches of Fig. l, depending on the relative position of points $0,1,2$, and 3 , which may be decided from the comparative values of $\mu$ and $\mu_{c}=$ $6.833 \cdots$, and from the comparative values of $\lambda \mu$ and $\left(\lambda_{\mu}\right)_{m},\left(\lambda_{\mu}\right)_{13}, \cdots$, as indicated in the caption of Fig. 1.

As is shown in Appendix A, for $\left(\phi_{s}, \gamma\right)$ in regions A, B, and C of Fig. 1, the number of roots of (3.1) with positive real parts is one, two, and zero, respectively. Therefore, the steady state is unstable in regions $A$ and $B$, and it is asymptotically stable in region $C$. Points of the upper curve correspond to bending points of the response curve, $v_{s}-\phi$. At points of the lower curve, a pair of complex conjugate roots of (3.1), $\omega= \pm i \Omega$, crosses the imaginary axis; at these points, a Hopf bifurcation occurs.

Now, it is easily seen that the response curve is as in one of the sketches of Fig. 3 , depending on the region of Fig. 2 to which the point $(\lambda \mu, \gamma)$ belongs. For example, if $\mu>\mu_{c}$ and $(\lambda \mu, \gamma) \in \mathrm{V}$ the neutral stability curves, $\gamma-\phi_{1}$, may be as in one of the sketches (g) or (h) of Fig. 1; in such a sketch, the response curve corresponds to a straight line $\gamma=$ constant, with $\max \left\{\gamma_{0}, \gamma_{3}\right\}<\gamma<\gamma_{2}$. Hence, the response curve is

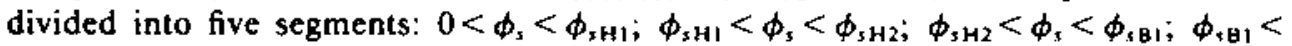
$\phi_{s}<\phi_{s B 2}$ and $\phi_{s B 2}<\phi_{s}$, in which the point $\left(\phi_{,}, \gamma\right)$ belongs to regions $C, B, C, A$, and $C$, respectively. Therefore, the response curve is as in sketch $V$ of Fig. 3, with two bending points, $\mathrm{B} 1$ and $\mathrm{B} 2$, and two Hopf bifurcations points, $\mathrm{H} 1$ and $\mathrm{H} 2$. In Fig. 3, no distinction is made in connection with the comparative values of $\phi_{\mathrm{B}_{1}}, \phi_{\mathrm{B}_{2}}, \phi_{\mathrm{H} 1}$, and $\phi_{\mathrm{H} 2}$; for example, in sketch $\mathrm{V}$, three additional possibilities could be considered, depending upon whether $\phi_{\mathrm{H} 1}<\phi_{\mathrm{H} 2}<\phi_{\mathrm{B} 2}, \phi_{\mathrm{H} 1}<\phi_{\mathrm{B} 2}<\phi_{\mathrm{H} 2}$ or $\phi_{\mathrm{B} 2}<\phi_{\mathrm{H}_{1}}<\phi_{\mathrm{H} 2}$. 


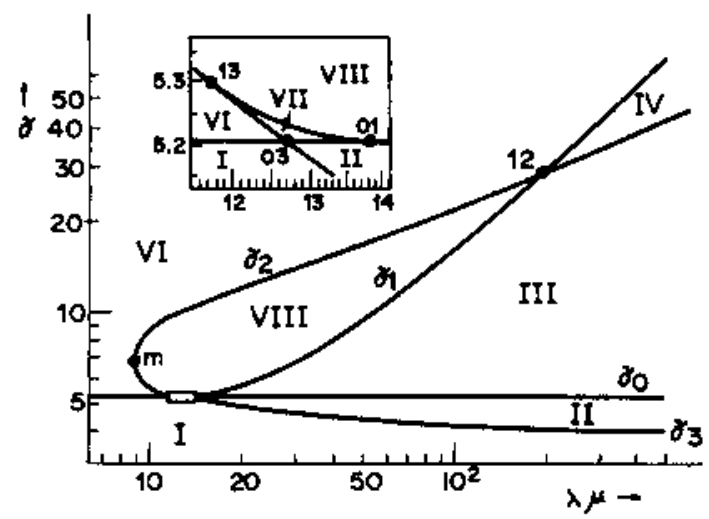

(a) $: \mu=2\left(\mu<\mu_{c}\right)$

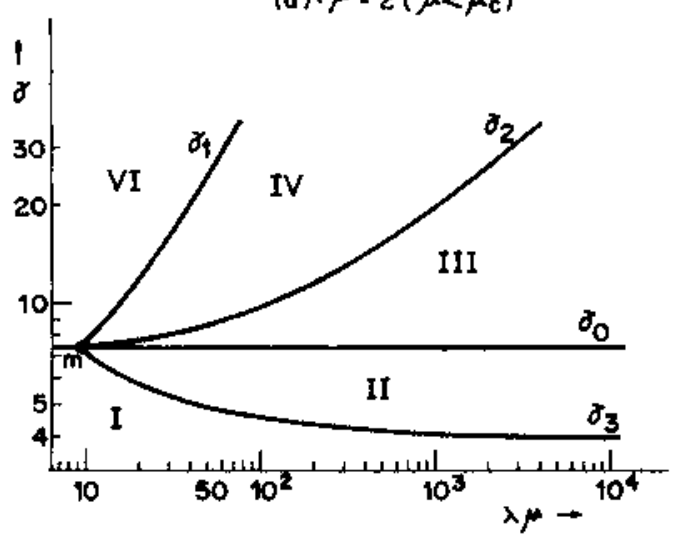

(b) $\mu=\mu_{c}=6.833 \ldots$

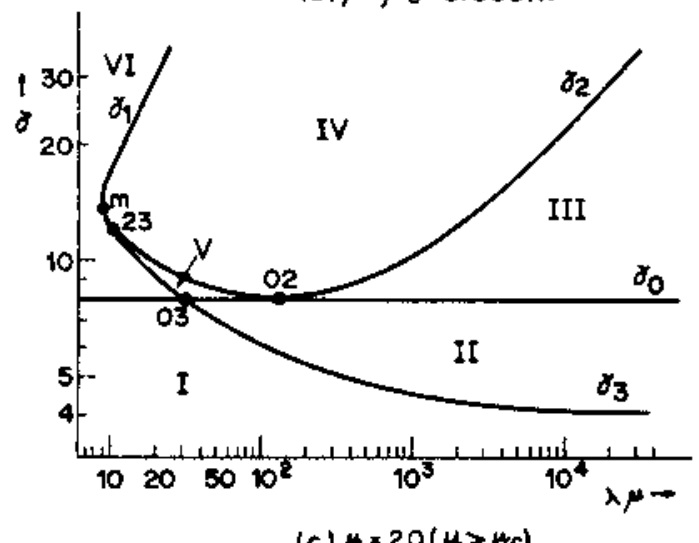

FIG. 2. Linear stability of Model 1 (slab geometry) for a fixed value of $\mu$. For $\mu<\mu_{c}$ (respectively, $\mu>\mu_{c}$ ), diagrams are qualitatively similar to diagram (a) (respectively, diagram (c)).

In order to get information about the size of the eight regions of Fig. 2, the coordinate of their vertices, as functions of $\mu$, are plotted in Fig. 4; observe that all vertices come together as $\mu \rightarrow \mu_{c}$, and that region VIII disappears as $\mu \rightarrow \mu_{c}$ from below.

3.2. Arbitrary shapes of the catalyst. We consider Model 1 and a bounded domain, $\Omega \subset \mathbb{R}^{p}(p=2$ or 3 ), whose boundary, $\partial \Omega$, is smooth and satisfies uniformly the interior and exterior sphere conditions: there exist two positive constants, $\rho_{1}$ and $\rho_{2}$, such that, 

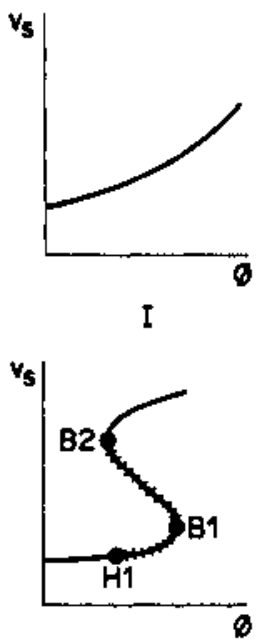

IV

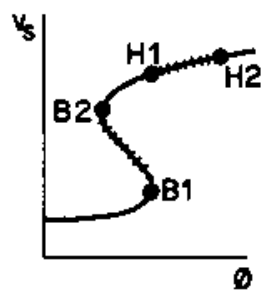

VII

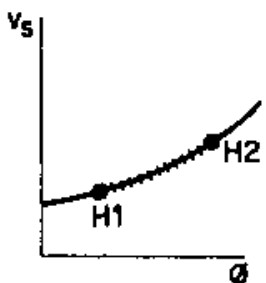

II

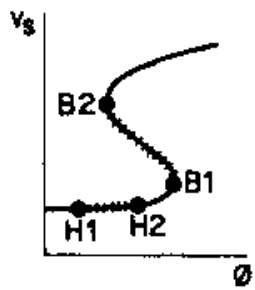

v

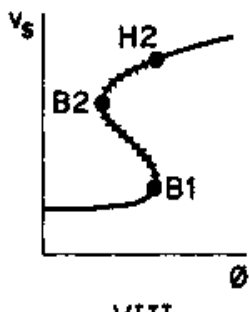

VIII

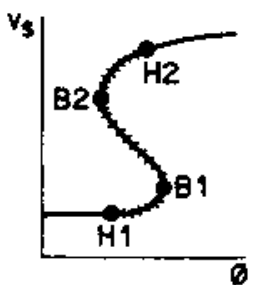

III

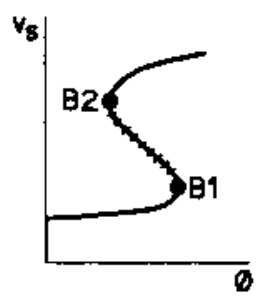

VI

F1G. 3. Response curves for Model 1 (slab geometry) and for Model 2. Asymptotically stable steady states. HH Unstable steady states.

for every point $q$ of $\partial \Omega$, two hyperspheres, $S_{1}$ and $S_{2}$, of radius $\rho_{1}$ and $\rho_{2}$, are tangent to $\partial \Omega$ at $q, S_{1}$ is included in $\Omega$, and $S_{2}$ and $\Omega$ are disjoint.

The steady state solutions of (1.3), (1.4) are given by

$$
\begin{aligned}
& \Delta u_{s}=\phi_{s}^{2} u_{s} \text { in } \Omega, \quad u_{s}=1 \text { on } \partial \Omega, \\
& v_{s}=1+\mu^{-1} \phi_{s}^{2} \int_{\Omega} u_{s} d x=1+\mu^{-1} \int_{\partial \Omega}\left(\frac{\partial u_{s}}{\partial n}\right) d s,
\end{aligned}
$$

in the terms of the parameter $\phi_{s}$, where

$$
\phi^{2}=\phi_{s}^{2} \exp \left(\gamma / v_{s}-\gamma\right) .
$$

Equation (3.7) uniquely defines a function $u_{s}=u_{s}\left(\phi_{s}, x\right)$, which is continuously differentiable in its dependence on $\phi_{s}$ and satisfies, for all $\phi_{s}>0$ (see Appendix B),

$$
\begin{aligned}
& 0<u_{s}\left(\phi_{s}, x\right)<1 \quad \text { for all } x \in \Omega, \\
& \frac{\partial u_{s}}{\partial n}<\phi_{s}+(p-1) / \rho_{2} \text { for all } x \in \partial \Omega .
\end{aligned}
$$

Then, the following inequalities are obtained from (3.8):

$$
v_{s}<1+\phi_{s}^{2} V_{\Omega} / \mu, \quad v_{s}<1+\left[\phi_{s}+(p-1) / \rho_{2}\right] S_{\Omega} / \mu .
$$



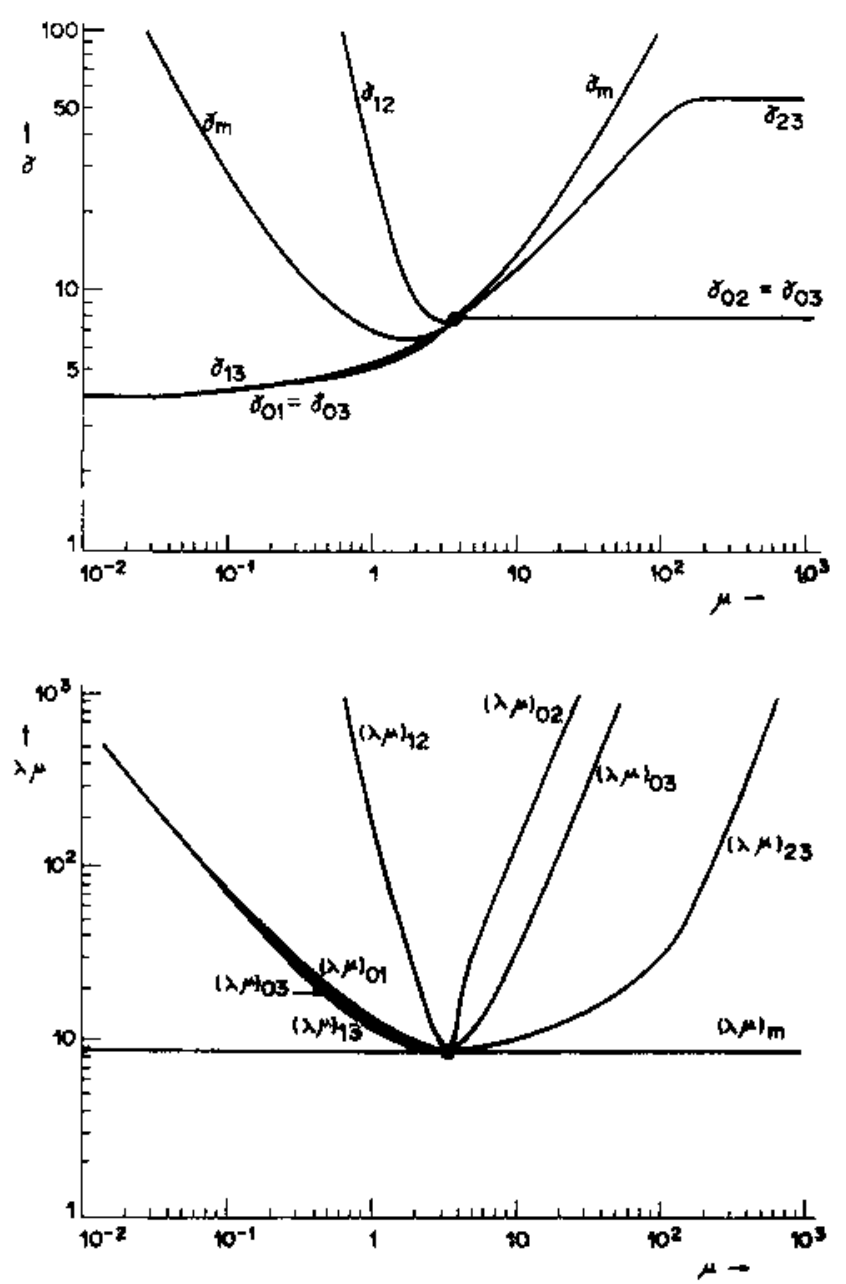

FIG. 4. Coordinates of the vertices of the regions of Fig. 2.

For fixed values of $\gamma$ and $\mu,(3.8),(3.9)$ define a $C^{1}$ response curve, $\phi_{s} \rightarrow\left(v_{s}, \phi\right)$. The derivatives $d v_{s} / d \phi_{s}$ and $d \phi / d \phi_{s}$ are

$$
\begin{aligned}
& \mu \frac{d v_{s}}{d \phi_{s}}=2 \mu\left(v_{s}-1\right) / \phi_{s}+\phi_{s}^{2} \int_{\Omega} \bar{u} d x=\int_{\partial \Omega}\left(\frac{\partial \bar{u}}{\partial n}\right) d s, \\
& 2 \phi^{-1} \frac{d \phi}{d \phi_{s}}=2 / \phi_{s}-\gamma v_{s}^{-2} \frac{d v_{s}}{d \phi_{s}},
\end{aligned}
$$

where $\bar{u}=\partial u_{s} / \partial \phi_{\mathrm{s}}$ is the unique solution of

$$
\Delta \vec{u}-\phi_{s}^{2} \bar{u}=2 \phi_{s} u_{s} \quad \text { in } \Omega, \quad \bar{u}=0 \quad \text { on } \partial \Omega,
$$

and satisfies, for all $\phi_{s}>0$ (see Appendix B),

$$
\begin{aligned}
& \bar{u}(x)<0 \text { for all } x \in \Omega, \\
& \frac{\partial \bar{u}}{\partial n}>2 \rho \phi_{s} / e\left[p+(2 p+1) \rho \phi_{s}\right] \text { for all } x \in \partial \Omega,
\end{aligned}
$$

where $\rho=\min \left\{\rho_{1}, \rho_{2} / 2(p-1)\right\}$. 
The following properties are easily obtained (3.13)-(3.17):

(i) $d v_{s} / d \phi_{s}>0$ for all $\phi_{\mathrm{s}}>0$.

(ii) $d \phi / d \phi_{s}>0$ if $\phi_{s}>0$ and

$$
\gamma\left(v_{s}-1\right) / v_{s}^{2} \leqq 1
$$

(iii) If $d \phi / d \phi_{s}>0$, then

$$
\gamma / v_{s}^{2}<\mu e\left[p+(2 p+1) \rho \phi_{s}\right] / \rho S_{\Omega} \phi_{s}^{2} .
$$

The linearized problem around the steady state has nontrivial solutions of the form $u-u_{s}=X(x) \exp (\omega t), v-v_{s}=Y \exp (\omega t)$, with $\operatorname{Re} \omega \geqq 0$, only if $(Y \neq 0$ and) $Z=X / Y$ and $\omega$ satisfy

$$
\begin{aligned}
& \Delta Z-\left(\omega+\phi_{s}^{2}\right) Z=\gamma v_{s}^{-2} \phi_{s}^{2} u_{s} \text { in } \Omega, \quad Z=0 \text { on } \partial \Omega, \\
& \omega / \lambda+\mu=\phi_{s}^{2} \int_{\Omega} Z d x+\gamma \mu\left(v_{s}-1\right) / v_{s}^{2} .
\end{aligned}
$$

In Appendix $B$, it is shown that:

(iv) If $(3.20),(3.21)$ has a nonreal eigenvalue such that $\operatorname{Re} \omega \geqq 0$, or if it has a double real eigenvalue $\omega \geqq 0$, then the following inequality must be satisfied:

$$
\left(\gamma / v_{s}^{2}\right)\left[\phi_{s}^{2} / \lambda+\mu-\gamma \mu\left(v_{s}-1\right) / v_{s}^{2}\right]+\left(\phi_{s}^{2}+k\right)^{3} / \lambda^{2} \phi_{s}^{4} V_{\Omega} \leqq 0,
$$

where

$$
k=\left(\omega_{p} / V_{\Omega}\right)^{2 / p} \text { and } \omega_{p}=2 \pi^{p / 2} / p \Gamma(p / 2) .
$$

For given values of $\gamma, \mu$ and $\phi_{s}$, and real values of $\omega \geqq 0,(3.20)$ uniquely defines a function $Z=Z(\omega, x)$, which is continuously differentiable in its dependence on $\omega$ and satisfies (see Appendix B)

$$
\begin{aligned}
& Z<0, \quad \frac{\partial Z}{\partial \omega} \geqq 0 \quad \text { for all } \omega \geqq 0 \text { and all } x \in \Omega, \\
& \int_{\Omega} Z(\omega, x) d x \rightarrow 0 \quad \text { as } \omega \rightarrow \infty
\end{aligned}
$$

Then, (3.21) may be written as

$$
\omega / \lambda+\mu=F(\omega),
$$

where the function $\omega \rightarrow F(\omega)$ is continuously differentiable and satisfies

$$
F^{\prime}(\omega) \geqq 0 \quad \text { for all } \omega \geqq 0, \quad F(\omega) \rightarrow \gamma \mu\left(v_{s}-1\right) / v_{s}^{2} \quad \text { as } \omega \rightarrow \infty .
$$

Furthermore, $\left(2 v_{s}^{2} / \gamma \phi_{s}\right) Z(0, x)=\partial u_{s} / \partial \phi_{s}$ (since it satisfies $(3.15)$ ), and

$$
F(0)=\mu-\left(\frac{\mu \phi_{s}}{\phi}\right) \frac{d \phi}{d \phi_{s}},
$$

as comes out from (3.13), (3.14), (3.21). Then:

(v) Equation (3.26) has (at least) a positive real root if the inequality (3.18) is not satisfied and $\lambda$ is sufficiently large (because then $\mu<F(\omega)$ for $\omega$ sufficiently large).

(vi) Equation (3.26) has no real positive roots if inequality (3.18) is satisfied (because then $\mu>F(\omega)$ for all $\omega \geqq 0$ ).

(vii) Equation (3.26) has (at least) a positive real root if $d \phi / d \phi_{s}<0$ (because then $\mu<F(0)$ and the second member of (3.26) is bounded). 
(viii) Equation (3.26) has no positive real roots if $d \phi / d \phi_{s}>0$ and $\lambda$ does not satisfy (3.22). To prove this property observe that, since $d \phi / d \phi_{s}>0$, the first member of (3.26) is larger than the second member for $\omega=0$. If $\lambda=\lambda_{1}$ does not satisfy (3.22), then (3.22) does not hold for any $\lambda<\lambda_{1}$. If, for $\lambda=\lambda_{1},(3.26)$ had a positive real root, then for some $\lambda=\lambda_{2} \leqq \lambda_{1},(3.26)$ would have a positive double real root (recall that $F$ is bounded); but this is not possible since $\lambda=\lambda_{2}$ does not satisfy (3.22) (property (iv)).

Now, from properties (i)-(viii) above, the following conclusions follow:

(A) The response curve, $v_{s}-\phi$, is either monotonous or $S$-shaped (with more than one $S$ possibly), as it comes out from property (i).

(B) If $\gamma \leqq 4$, then (3.18) holds for all $v_{s} \geqq 1$ (i.e., for all $\phi_{s} \geqq 0$ ), and the response curve is monotonous (property (ii)).

(C) If $\gamma>4$, let $v_{s 1}$ and $v_{s 2}$ be the solutions of the quadratic equation $\gamma\left(v_{s}-1\right) / v_{s}^{2}=$ 1 , let $\phi_{s 1}$ and $\phi_{s 2}$ be the corresponding values of $\phi_{s}$, and let points 1 and 2 be the corresponding points of the response curve. For $0<\phi_{s}<\phi_{s 1}$ and for $\phi_{s 2}<\phi_{s}$, inequality (3.18) holds and $d \phi / d \phi_{s}>0$ (property (ii)); then, if the response curve is not monotonous, points 1 and 2 belong to the lower and upper branches of the response curve; furthermore, the segments $0<\phi_{s}<\phi_{s 1}$ and $\phi_{s 2}<\phi_{s}$ correspond to asymptotically stable steady states (properties (iv) and (vi)). On the other hand, in the segment $\phi_{s 1}<\phi_{s}<\phi_{s 2}$, inequality (3.18) is not satisfied (and the corresponding steady states are unstable) if $\lambda$ is sufficiently large (property (v)).

(D) Finally, from properties (iii), (iv), (vii), and (viii), it comes out that if inequalities (3.19) and (3.22) are not satisfied simultaneously for any positive value of $\phi_{s}$, then a steady state is asymptotically stable or unstable according to whether $d \phi / d \phi_{s}>0$ or $d \phi / d \phi_{s}<0$ at the corresponding point of the response curve. This simple geometrical criterion applies, in particular, if one of the following inequalities is satisfied:

$$
\begin{aligned}
& \gamma \leq 2+2 \sqrt{1+16 k / \lambda^{2} \mu V_{\Omega}}, \\
& \gamma \geq \frac{\lambda \mu \rho_{2}^{2} S_{\Omega}^{2}+\left[\rho_{2} \mu+(p-1) S_{\Omega}\right]^{2}}{4 \lambda^{2} \mu^{3} \rho_{2}^{2} V_{\Omega}} k\left[H_{1}^{2}\left(H_{1}+2 H_{2}+\sqrt{\left.H_{1}^{2}+4 H_{1} H_{2}-4\right)^{2}}-27\right],\right. \\
& \lambda \mu<2 k \rho S_{\Omega} / V_{\Omega} e\left[\sqrt{k} \rho(2 p+1)+\sqrt{p^{2}+k \rho^{2}(2 p+1)^{2}}\right],
\end{aligned}
$$

where

$$
H_{1}=e \lambda \mu V_{\Omega} p / k \rho S_{\Omega}, \quad H_{2}=\sqrt{k} \rho(2 p+1) / p
$$

as may be seen when using (3.12).

4. Linear stability for Model 2. The steady state solutions of (1.6)-(1.8) are

$$
U_{s}=\exp \left(\Phi_{s} \xi\right) /\left(1+\Phi_{s}\right), \quad v_{s}=\left[m+(1+m) \Phi_{s}\right] / m\left(1+\Phi_{s}\right),
$$

in terms of the parameter $\Phi_{s}$, which is given by

$$
\Phi^{2}=\Phi_{s}^{2} \exp \left(\gamma / v_{s}-\gamma\right) \text {. }
$$

The linearized problem around the steady state has nontrivial solutions if and only if

$$
\begin{aligned}
& \omega(\omega+l m)=\frac{\gamma l m^{2} \Phi_{s}\left(1+\Phi_{s}\right)}{\left[m+(1+m) \Phi_{s}\right]^{2}}\left(\omega-\phi_{s}^{2}+\frac{\Phi_{s}^{3}\left(1+\Phi_{s}\right)}{\omega+\Phi_{s}^{2}+\sqrt{\omega+\Phi_{s}^{2}}}\right) \\
& \gamma=\frac{2\left[m+(1+m) \Phi_{s}\right]^{2}}{m \Phi_{s}}
\end{aligned}
$$


for $\omega \neq 0$ and $\omega=0$, respectively. Neutral stability points are as sketched in Fig. 5 , where the upper curve is given by (4.2). The coordinates of point 0 are

$$
\Phi_{s 0}=m /(m+1), \quad \gamma_{0}=8(m+1) .
$$

When $\omega$ is replaced by $i \Omega$ in (4.1), the following equations result

$$
\begin{aligned}
& \gamma=\frac{a\left[m+(1+m) \Phi_{s}\right]^{2}\left[2 \operatorname{lm}\left(2 \Phi_{s}^{2}+a\right)+a(1+a)\left(4 \Phi_{s}^{2}-a^{2}\right)\right]}{2 l m^{2} \Phi_{s}\left(1+\Phi_{s}\right)\left(a^{2}-2 \Phi_{s}^{2}\right)}, \\
& \frac{\Omega\left(2 \Phi_{s}^{2}+a\right)+\operatorname{lm} b(a+1)}{\Omega b(a+1)-\operatorname{lm}\left(2 \Phi_{s}^{2}+a\right)}=\frac{\Phi_{s}^{2}\left(2 \Phi_{s}-a\right)-\Omega b(a+1)}{\Omega a-\Phi_{s}^{2} b}
\end{aligned}
$$

where $a>0$ and $b \geqq 0$ are given by

$$
a^{2}=2\left(\Phi_{s}^{2}+\sqrt{\Omega^{2}+\Phi_{s}^{4}}\right), \quad b^{2}=2\left(-\Phi_{s}^{2}+\sqrt{\Omega^{2}+\Phi_{s}^{4}}\right) .
$$

For $0<\Phi_{s} \leqq \Phi_{s 1},(4.4)$ defines a nonnegative function

$$
\Omega=\Omega\left(\operatorname{lm}, \Phi_{s}\right),
$$

which is such that $\Omega\left(l m, \Phi_{s 1}\right)=0, \Omega\left(l m, \Phi_{s}\right)>0$ for $0<\Phi_{s}<\Phi_{s 1}$, where $\Phi_{s 1}$ is the unique solution of the equation

$$
l m=4 \Phi_{s}^{2}\left(1+\Phi_{s}\right) /\left[1+\Phi_{s}+2\left(1+2 \Phi_{s}\right)^{2}\right]
$$

The lower curve in the sketches of Fig. 5 is given by (4.3), with $\Omega$ as given by (4.5). The coordinates of point 1 are $\left(\Phi_{s 1}, \gamma_{1}\right)$, where $\gamma_{1}$ is given by (4.2), with $\Phi_{s}=\Phi_{s 1}$. Point 2 (when it exists) is the unique minimum of the curve; its coordinates must be computed numerically.

As in Appendix A, the argument principle shows that in regions $\mathrm{A}, \mathrm{B}$, and $\mathrm{C}$ of Fig. 5, the number of roots of (4.1) with positive real parts is one, two, and zero, respectively. Then, the steady state is unstable in regions $A$ and $B$, and it is asymptotically stable in region $C$. Points of the upper curve of Fig. 5 are bending points of the response curve, and points of the lower curve are Hopf bifurcation points.

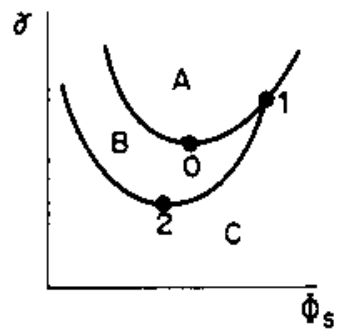

(a)

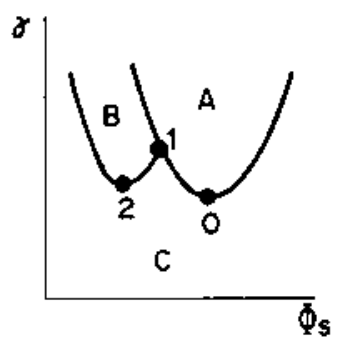

(c)

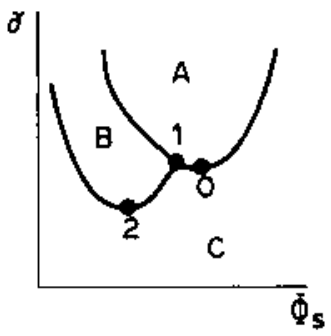

(b)

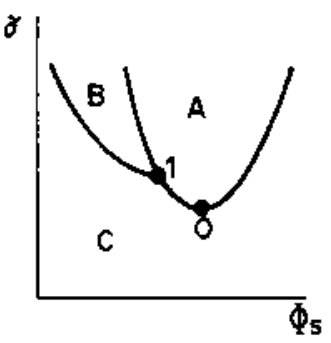

(d)

FIG. 5. Curves of neutral stability points of Model 2 for fixed volues of $I$ and $m$. (a) (im $\rangle_{01}<\mathrm{im}$; (b) $(l m)_{02}<l m<(l m)_{01}$; (c) $(l m)_{02}<l m<(l m)_{02}$; (d) $0<i m<(l m)_{12}$. 


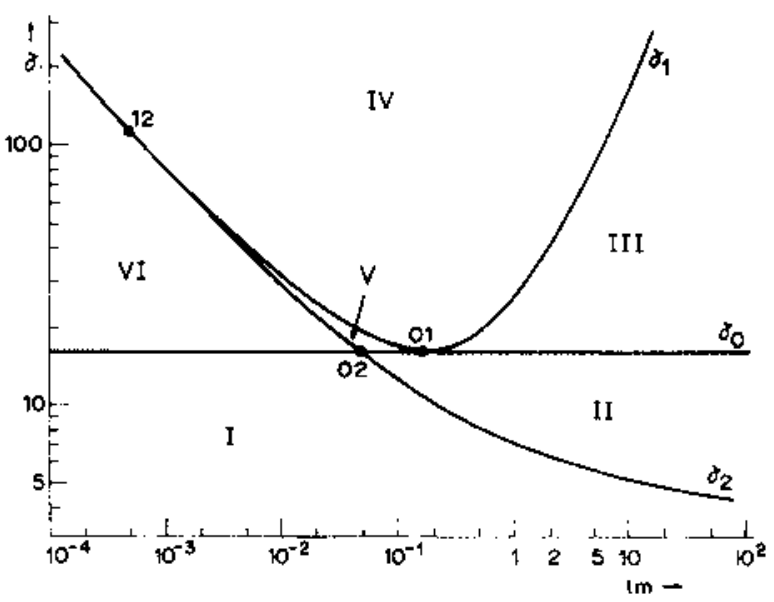

FIG. 6. Linear stability of Model 2 for $m=1$.
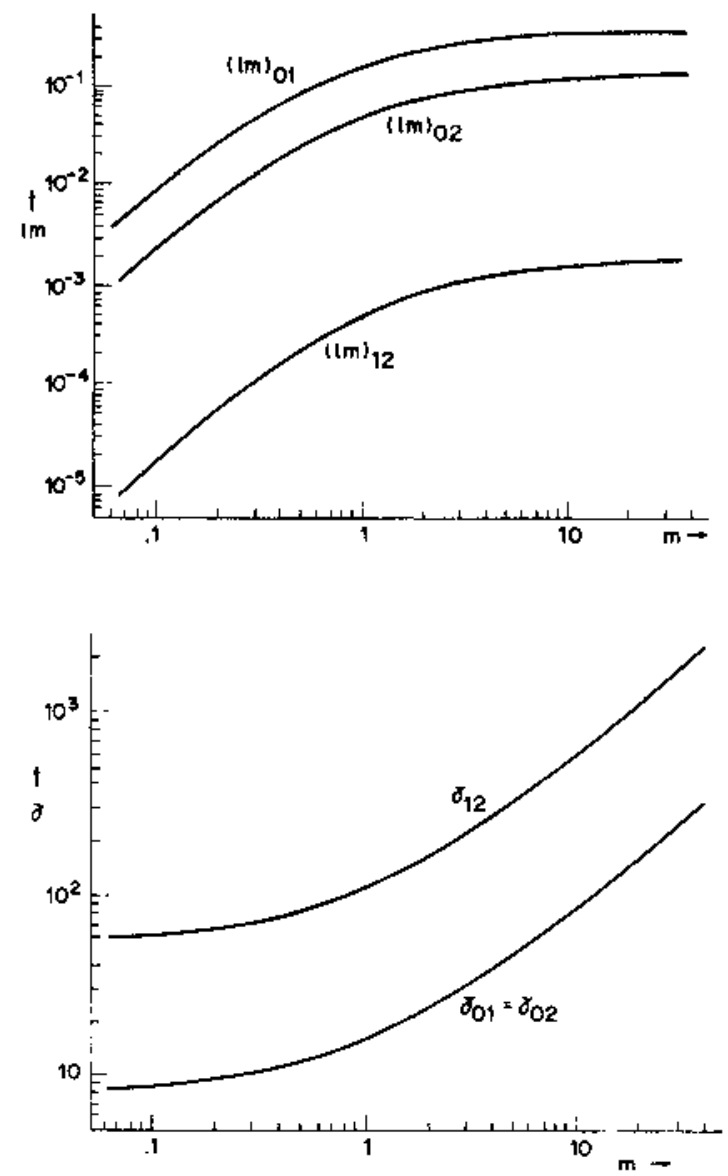

FiG. 7. Coordinates of the vertices of the regions of Fig. 6. 
For a fixed value of $m$, as $l$ increases, the upper curve of Fig. 5 remains constant, $\Phi_{s 1}$ increases, and any point of the lower curve moves down. For a fixed value of $m$, the functions $\gamma_{i}=\gamma_{i}(\mathrm{Im})$, for $i=0,1$, and 2, are qualitatively similar to those plotted in Fig. 6. The asymptotic behavior of $\gamma_{1}$ and $\gamma_{2}$ is given by: $\sqrt{I m} \gamma_{1} \rightarrow 4 m / \sqrt{3}$ as $l m \rightarrow 0$, $\gamma_{1} / l m \rightarrow 4(1+m)^{2} / m$ as $l m \rightarrow \infty ; \gamma_{2} \rightarrow 4$ if $m \leqq 1, \gamma_{2} \rightarrow(1+m)^{2} / m$ if $m>1$, as $l m \rightarrow \infty$. The coordinates of the vertices of the six regions of Fig. 6 are plotted, versus $m$, in Fig. 7.

As in $\$ 2$, the response curve is as in one of the sketches I-VI of Fig. 3, depending on the region of Fig. 6 to which the point $(l m, \gamma)$ belongs.

4.1. The limit $m \rightarrow 0$. In the distinguished limit $\Phi_{s}^{2} \sim m^{2} \sim \Omega \sim l m \rightarrow 0, \gamma_{0}=8$ is constant to leading order. The function (4.5) is given, in first approximation, by

$$
\frac{\Omega}{l m}=\frac{\eta^{2}(2+\eta) \sqrt{1-\eta^{2}}}{(1+\eta)\left(4-2 \eta^{2}-\eta^{3}\right)}, \quad \frac{\Phi_{s}^{2}}{l m}=\frac{\eta^{4}(2+\eta)}{2(1+\eta)\left(4-2 \eta^{2}-\eta^{3}\right)},
$$

in terms of the parameter $\eta$, for $0 \leqq \eta \leqq 1$, where

$$
\eta^{2}=2 \Phi_{s}^{2} /\left(\Phi_{s}^{2}+\sqrt{\Omega^{2}+\Phi_{s}^{4}}\right)
$$

and (4.3) may be written, in first approximation, in the form

$$
\gamma=\frac{4\left(m+\Phi_{s}\right)^{2}}{m \sqrt{\operatorname{lm}}} \frac{\sqrt{1 \mp \eta}\left(2-\eta^{2}\right)}{\eta^{2} \sqrt{4+2 \eta} \sqrt{4-2 \eta^{2}-\eta^{3}}} .
$$

Then, to leading order, $\gamma_{1}$ is given by

$$
\gamma_{1}=4(\sqrt{l m} / \sqrt{3} m)(m / \sqrt{l m}+\sqrt{3} / 2)^{2},
$$

and $\gamma_{3}$ is given by (4.8), with $\Phi_{s}$ as in (4.7) and $m / \sqrt{l m}$ as given by

$$
\frac{m}{\sqrt{l m}}=\frac{\Phi_{s}}{\sqrt{l m}}\left(1+\frac{4 \eta^{3}(2+\eta)(1+\eta)\left(6-\eta^{2}\right)}{3 \eta^{7}+12 \eta^{6}+2 \eta^{5}-56 \eta^{4}-92 \eta^{3}-16 \eta^{2}+88 \eta+64}\right) .
$$

A plot of the linear stability diagrams of Fig. 6 in this limit is given in Fig. 8 , where $(l m)_{01} / m^{2}=4 / 3,(l m)_{02} / m^{2}=0.3138 \cdots,(l m)_{12} / m^{2}=4 / 1875, \gamma_{01}=\gamma_{02}=8$, and $\gamma_{12}=2 \cdot 26^{2} / 25$.

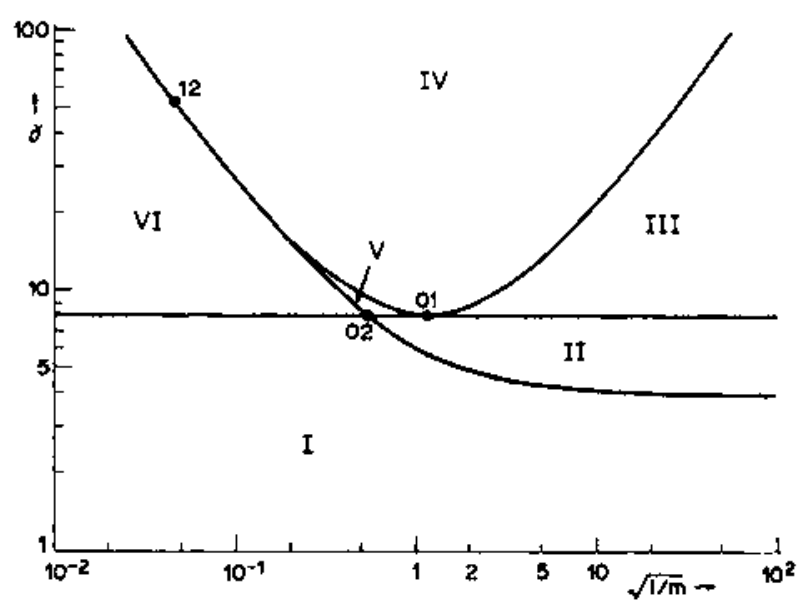

F3G. 8. Linear stability of Model 2 in the limit $m \rightarrow 0$. 
4.2. The limit $m \rightarrow \infty$. In the distinguished limit $\Phi_{s}^{2} \sim \Omega \sim l m \sim 1, m \rightarrow \infty$, (4.2) and (4.3) may be written, in first approximation, as

$$
\gamma / m=2\left(1+\Phi_{s}\right)^{2} / \Phi_{s}
$$

$$
\gamma / m=a\left(1+\Phi_{s}\right)\left[2 \operatorname{lm}\left(2 \Phi_{s}^{2}+a\right)+a(1+a)\left(4 \Phi_{s}^{2}-a^{2}\right)\right] / 2 \operatorname{lm} \Phi_{s}\left(a^{2}-2 \Phi_{s}^{2}\right)
$$

Therefore, to leading order, $\gamma_{0} / m=8$ and $\gamma_{1} / m$ is given by (4.9), with $\Phi_{s}=\Phi_{s 1}$, where $\Phi_{s 1}$ is the unique root of (4.6). $\gamma_{2} / m$ must be computed numerically, as the unique minimum of the function $\Phi_{s} \rightarrow \gamma / m$, defined by (4.5) and (4.10). A plot of the linear stability diagram of Fig. 6 in this limit is given in Fig. 9, where $(\mathrm{lm})_{12}=0.001860 \cdots$, $(l m)_{02}=0.1430 \cdots,(l m)_{01}=0.4, \gamma_{t 2} / m=55.63 \cdots$, and $\gamma_{02} / m=\gamma_{01} / m=8$.

5. Hopf bifurcation. Let us now consider local Hopf bifurcation at points $\mathrm{H} 1$ and H2 of Fig. 3. For the sake of brevity, only Model 2 will be considered; a similar analysis applies to Model 1.

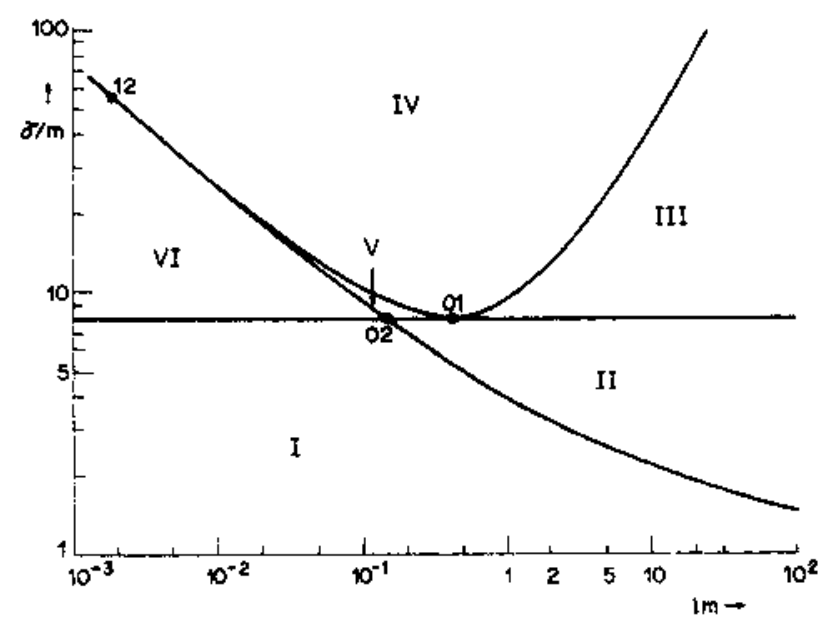

FiG, 9. Linear stability of Model 2 in the limit $m \rightarrow \infty$.

Let $\alpha$ be a parameter (to be chosen later) giving a first approximation of the size of the bifurcated periodic orbits, and let $\Phi_{s \mathrm{H}}$ be $\Phi_{s \mathrm{Hi}}$ or $\Phi_{s \mathrm{H} z}$. When using $\Phi_{s}$ as bifurcation parameter, it is expanded in the form

$$
\Phi_{s}=\Phi_{s H}+B \alpha^{2}+\cdots
$$

Then, if $\Phi_{s \mathrm{H}}=\Phi_{s \mathrm{H} 1}$ (respectively, $\Phi_{s \mathrm{H}}=\Phi_{s \mathrm{H} 2}$ ), the bifurcation is subcritical or supercritical depending on whether $B<0$ or $B>0$ (respectively, $B>0$ or $B<0$ ).

In order to simplify the algebra of the analysis below, we shall use $\gamma$ as bifurcation parameter. To this end, $\gamma$ will be expanded as

$$
\gamma=\gamma_{\mathrm{c}}+\boldsymbol{A} \alpha^{2}+\cdots
$$

If the lower curve of Fig. 5 is given by $\gamma=f\left(\Phi_{s}\right)$ (then $\gamma_{c}=f\left(\Phi_{s \mathrm{H}}\right)$ ), $f^{\prime}\left(\Phi_{s \mathrm{H}}\right)$ is negative or positive depending on whether $\Phi_{s \mathrm{H}}=\Phi_{s \mathrm{H}_{1}}$ or $\Phi_{s \mathrm{H}}=\Phi_{\mathrm{sH}_{2}}$. It is easily seen also that $B=-A / f^{\prime}\left(\Phi_{k H}\right)$. Therefore, bifurcation at $\Phi_{v H}$ is subcritical if $A<0$, and it is supercritical if $A>0$. 
For $0<\Phi_{s \mathrm{H}}<\Phi_{s \mathrm{H} 1}$, the pair of roots of (4.1) that (for varying $\gamma$ and fixed $\Phi_{s}=\Phi_{s \mathrm{H}}$, $l$, and $m$ ) crosses the imaginary axis of the complex plane at $\gamma=\gamma_{c}$, satisfies the transversality condition

$$
d(\operatorname{Re} \omega) / d \gamma \neq 0, \quad \operatorname{Im} \omega=\Omega_{c} \neq 0,
$$

at $\gamma=\gamma_{c}$, while the remaining roots of (4.1) have strictly negative real parts. In addition, the solution of (1.6)-(1.8) satisfies the smoothness hypothesis of Hopf bifurcation theorem (Marsden and McCracken [9], Hassard et al. [10]), as is seen by means of standard theory on semilinear parabolic equations (see, e.g., Hassard et al. [10], Henry [11]). Hence, the Hopf bifurcation theorem shows that, for $\gamma$ sufficiently close to $\gamma_{c}$ (i.e., for $\gamma$ as given by (5.1) and $\alpha$ sufficiently small), there exists a periodic solution of (1.6)-(1.8), of period

$$
T(\alpha)=2 \pi / \Omega \quad \text { with } \Omega=\Omega_{c}+\alpha \Omega_{1}+\alpha^{2} \Omega_{2}+\cdots
$$

which may be written in the form

$$
U=U_{s}+\alpha U_{0}+\alpha^{2} U_{1}+\alpha^{3} U_{2}+\cdots, \quad v=v_{s}+\alpha v_{1}+\alpha^{2} v_{2}+\cdots .
$$

Furthermore, bifurcated orbits are unstable if bifurcation is subcritical $(A<0)$, and they are orbitally, asymptotically stable if bifurcation is supercritical $(A>0)$.

The coefficients of the expansions (5.1)-(5.3) may be calculated systematically by means of known recursive formulae (Hassard et al. [10], Kielhöfer [12]), via reduction to the center manifold, that also allows us to analyze degenerate cases in which the transversality condition is not satisfied. Nevertheless, due to the inherent algebraic complexity of the problem (1.6)-(1.8), we shall use a more direct (although a less systematic) approach, and we shall not try to analyze degenerate cases in detail. Some conclusions about the nature of degenerate cases may be drawn from the results for neighboring nondegenerate ones, by means of simple normal forms arguments (Golubitsky and Langford [13], Guckenheimer [14]), when taking into account that the flow defined by (1.6)-(1.8) is essentially two-dimensional near a bifurcation point, since it may be reduced (locally) to a center manifold for sufficiently large time.

In order to look for $2 \pi$ periodic solutions, we introduce the new time variable $s=2 \pi \tau / T$, where the period $T$ is given by (5.2). Then, $A, \Omega_{i}, U_{i}$, and $v_{i}$, the coefficients of the expansions (5.1)-(5.3), may be calculated from the set of recursive problems which results when the expansions (5.1)-(5.3) are inserted into (1.6)-(1.8), and the coefficient of each power of $\alpha$ is set to zero. Such problems are of the form

$$
\begin{aligned}
& \Omega_{c} \frac{\partial U_{i}}{\partial s}-\frac{\partial^{2} U_{i}}{\partial \xi^{2}}+\Phi_{s H}^{2} U_{i}+\gamma_{c} \Phi_{s \mathrm{H}}^{2} U_{s} v_{i} / v_{s}^{2}=f_{i} \quad \text { in }-\infty<\xi<0, \\
& U_{i}=0 \quad \text { at } \xi=-\infty, \quad \frac{\partial U_{i}}{\partial \xi}+U_{i}=0 \text { at } \xi=0, \\
& \Omega_{c} \frac{d v_{i}}{d s}+\operatorname{lm}\left[1-\gamma_{c}\left(v_{s}-1\right) / v_{s}^{2}\right] v_{\mathrm{i}}-l \Phi_{s \mathrm{H}}^{2} \int_{-\infty}^{0} U_{i} d \xi=g_{i},
\end{aligned}
$$

where $f_{0} \equiv 0$ and $g_{0} \equiv 0$. In addition, we impose the periodicity conditions

$$
U_{i}(0, \xi) \equiv U_{i}(2 \pi, \xi), \quad v_{i}(0)=v_{i}(2 \pi),
$$

and an additional condition in order to define the parameter $\alpha$; this condition, which is quite arbitrary, may be, for example, $v(0)=v_{s}+2 \alpha v_{s}^{2} / \gamma_{c}, v^{\prime}(0)=0$, or $v_{0}(0)=2 v_{s}^{2} / \gamma_{c}$, $v_{i}(0)=0$ for $i \geqq 1 ; v_{i}^{\prime}(0)=0$ for $i \geqq 0$. 
It is easily seen that (5.4)-(5.6) has a solution satisfying (5.7) only if the second members of (5.4) and (5.6) satisfy the solvability condition

$$
\int_{0}^{2 \pi} \exp (-i s)\left(\int_{-\alpha}^{0} X^{*} f_{i} d \xi+Y^{*} g_{i}\right) d s=0,
$$

where

$$
X^{*}=l \Phi_{s \mathrm{H}}^{2} Y^{*}\left(1-\exp \left(\sqrt{\Phi_{s \mathrm{H}}^{2}+i \Omega_{c}} \xi\right) /\left(1+\sqrt{\Phi_{s \mathrm{H}}^{2}+i \Omega_{c}}\right)\right) /\left(\Phi_{s \mathrm{H}}^{2}+i \Omega_{c}\right), \quad Y^{*} \neq 0
$$

is a nontrivial solution of the adjoint linearized problem:

$$
\begin{aligned}
& \frac{d^{2} X^{*}}{d \xi^{2}}-\left(\Phi_{s \mathrm{H}}^{2}+i \Omega_{c}\right) X^{*}+1 \Phi_{s \mathrm{H}}^{2} Y^{*}=0 \quad \text { in }-\infty<\xi<0, \\
& X^{*} \text { bounded at } \xi=-\infty, \quad \frac{d X^{*}}{d \xi}+X^{*}=0 \quad \text { at } \xi=0, \\
& \gamma_{c} v_{s}^{-2} \Phi_{s \mathrm{H}}^{2} \int_{-x}^{0} U_{s} X^{*} d \xi+i \Omega_{c} Y^{*}+\operatorname{lm}\left[1-\gamma_{s}\left(v_{s}-1\right) / v_{s}^{2}\right] Y^{*}=0 .
\end{aligned}
$$

When $U_{0}$ and $v_{0}$ are inserted into the second terms of (5.4) and (5.6), it is easily seen that the solvability condition (5.8) is satisfied for $i=1$ only if $\Omega_{1}=0$. Then $U_{1}$ and $v_{1}$ are easily calculated; when they are inserted into the second members of (5.4) and (5.6), and the solvability condition for $i=2$ is applied, a pair of linear equations is obtained to calculate $\Omega_{2}$ and $A$. The solution of these equations yields

$$
A=\gamma\left[C_{1}\left(1+C_{3}\right)+D_{1} D_{3}\right] /\left[\operatorname{lm}\left(1+C_{3}\right)+\Omega D_{3}\right],
$$

where

$$
\begin{aligned}
& C_{1}=\Phi_{s}^{2}\left[C_{3}\left(1+E_{1}\right)-E_{1}-2 C_{2}-2 E_{4}\right]+E_{2}+E_{3}\left(C_{2}+E_{1}\right)+2 \Omega\left(1-E_{4}\right), \\
& D_{1}=\Phi_{s}^{2}\left[D_{3}\left(1+E_{1}\right)-2 D_{2}+\left(2 \Phi_{s}^{2}-E_{3}\right) / 2 \Omega\right] \\
& +\Omega\left[2\left(E_{4}-1\right)\left(C_{2}+E_{1}\right)-2 E_{4}+3 E_{4}^{2}\right]+D_{7} E_{3} \text {, } \\
& C_{2}=\left(C_{4} C_{5}-D_{4} D_{5}\right) /\left(C_{5}^{2}+D_{5}^{2}\right), \quad D_{2}=\left(C_{4} D_{5}+C_{5} D_{4}\right) /\left(C_{5}^{2}+D_{5}^{2}\right), \\
& C_{3}+i D_{3}=I \Phi_{s}^{3}\left(D_{6}-i C_{6}\right)\left(1+C_{8}+i D_{8}\right) / \gamma\left(1+\Phi_{8}\right) \Omega E_{4}^{2} \text {, } \\
& C_{4}=\Omega^{2}\left[\Phi_{s}+2 y m\left(1+\Phi_{s}\right) E_{4}^{2}\left(E_{4}-1\right)\right]+\Phi_{s}^{5}\left(C_{7}-2 C_{8}\right)-\Phi_{s}^{3} \Omega D_{7} / 2 \text {, } \\
& D_{4}=2 \gamma\left(1+\Phi_{s}\right) \Omega^{3} E_{4}^{2}\left(2 E_{4}-1\right) / 1+\Phi_{s}^{5}\left(D_{7}-2 D_{8}\right)+\Phi_{s}^{3} \Omega C_{7} / 2 \text {, } \\
& C_{5}=2 \Omega^{2}\left[\Phi_{s}-\gamma m\left(1+\Phi_{s}\right) E_{4}^{2}\right]+\Phi_{s}^{3} \Omega D_{7}, \quad D_{5}=4 \gamma\left(1+\Phi_{s}\right) \Omega^{3} E_{4}^{2} / l+\Phi_{s}^{3} \Omega C_{7}, \\
& C_{6}+i D_{6}=\frac{1+2 \sqrt{\Phi_{s}^{2}+i \Omega}}{2\left(\Phi_{s}^{2}+i \Omega\right)\left(1+\sqrt{\Phi_{s}^{2}+i \Omega}\right)}-\frac{1+\Phi_{s}+\sqrt{\Phi_{s}^{2}+i \Omega}}{\Phi_{s}\left(1+\Phi_{s}\right)\left(\Phi_{s}+\sqrt{\Phi_{s}^{2}+i \Omega}\right)}, \\
& C_{7}+i D_{7}=\Phi_{s}\left(1+\Phi_{s}\right) /\left(1+\sqrt{\Phi_{s}^{2}+2 i \Omega}\right) \sqrt{\Phi_{s}^{2}+2 i \Omega}-1 \text {, } \\
& C_{8}+i D_{8}=\Phi_{s}\left(1+\Phi_{s}\right) /\left(1+\sqrt{\Phi_{s}^{2}+i \Omega}\right) \sqrt{\Phi_{s}^{2}+i \Omega}-1 \text {, } \\
& E_{1}=\frac{4 \gamma m\left(1+\Phi_{s}\right)^{2} E_{4}^{2}\left(E_{4}-1\right)+\Phi_{s}\left(3+4 \Phi_{s}\right)+4 \Phi_{s}^{5}\left(1+\Phi_{s}\right) C_{8} / \Omega^{2}}{\Phi_{s}-2 \gamma m\left(1+\Phi_{s}\right)^{2} E_{4}^{2}}, \\
& E_{2}=\operatorname{lm}\left(1-3 E_{4}+3 E_{4}^{2}\right), \quad E_{3}=\operatorname{lm}\left(2 E_{4}-3\right)+2 l \Phi_{s} / \gamma\left(1+\Phi_{s}\right) E_{4}^{2}, \\
& E_{4}=\left[m+(1+m) \Phi_{s}\right] / \gamma m\left(1+\Phi_{s}\right) \text {. }
\end{aligned}
$$

Here, the suffixes $H$ and $c$ have been dropped from $\Phi_{s}, \Omega$, and $\gamma$; also $\gamma$ and $\Omega$ are given by (4.3) and (4.5). Therefore, (5.9) defines, for $0<\Phi_{s}<\Phi_{s 1}$, a function of the type $A=A\left(l m, m, \Phi_{s}\right)$, which allows us to know whether Hopf bifurcation (at points 
$\mathrm{H} 1$ and $\mathrm{H} 2$ ) is subcritical or supercritical. Numerical computations are summarized in Figs. 10 and 11 ; in the regions $\mathrm{IIa}, \cdots, \mathrm{Vb}$ (respectively, I and VI) of the diagrams sketched in Fig. 10, the response curves are as sketched in Fig. 11 (respectively, in Fig. 3). Observe that the diagrams of Fig. 10 are partitions of the diagram of Fig. 6.

The dynamic response of the catalyst in a quasi-steady operation (i.e., when the parameters are varied slowly enough) near a point of neutral stability, depends on whether Hopf bifurcation is sub- or supercritical at this point. In the supercritical (respectively, subcritical) case, as the steady state becomes unstable, neighboring initial conditions lead to oscillations of small amplitude (respectively, to some other attractor,

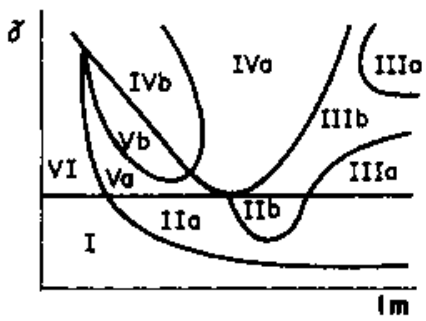

(a) $0<m<.0392 \ldots$

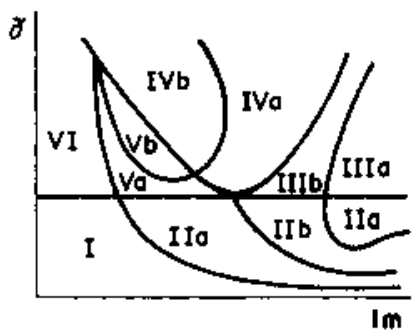

(c) $.321 \ldots<m<.349 \ldots$

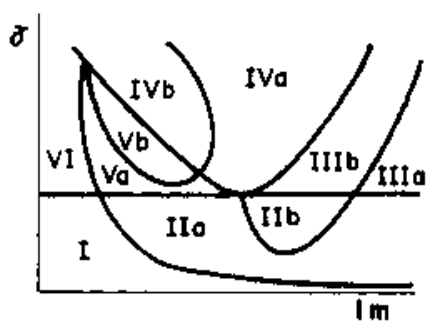

(e) $.515 \ldots<m<.595 \ldots$

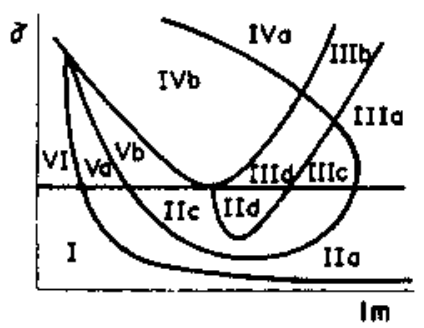

(o) $1.736 \ldots<m$.

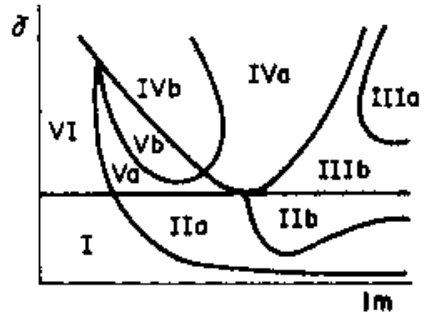

(b) $.0392 \ldots<m<.321 \ldots$

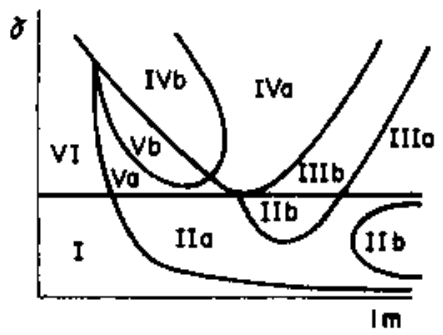

(d) $.349 \ldots<m<.515 \ldots$

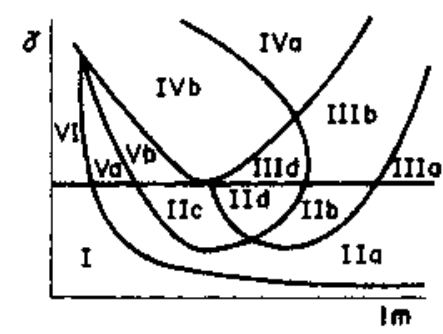

$(f) .595 \ldots<m<1.736 \ldots$

FIG. 10. Hopf bifurcation for Model 2. 


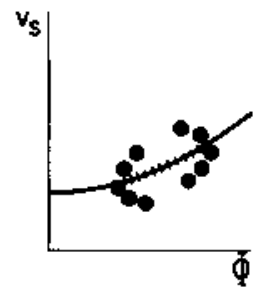

IIo

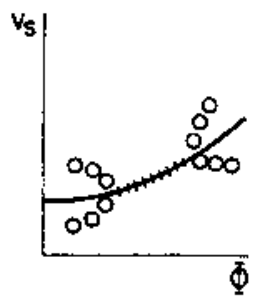

IId

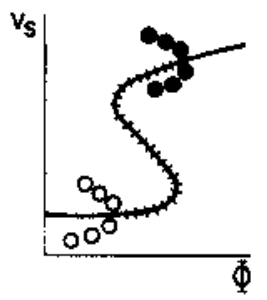

IIIC

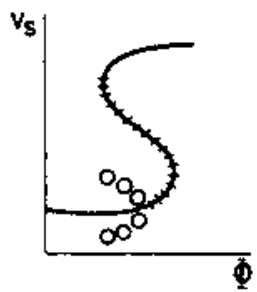

IVD

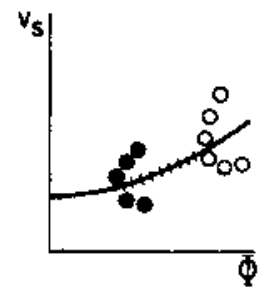

IIb

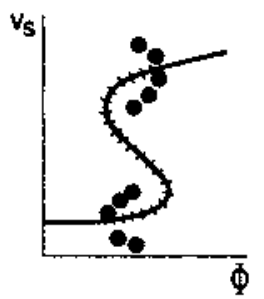

IIIO

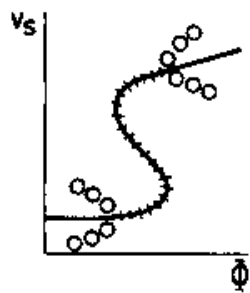

III

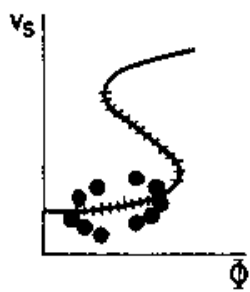

Vo

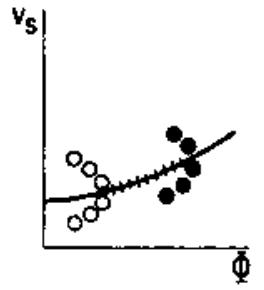

IIC

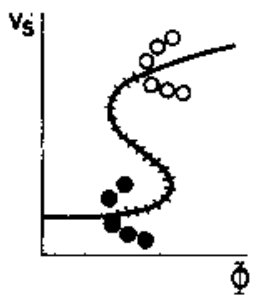

IIID

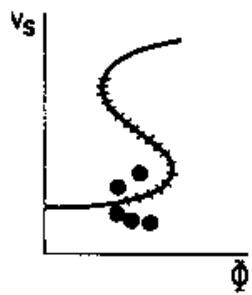

IVa

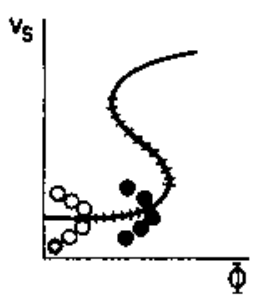

Vb

FIG. 11. Response curves for Model 2. -.. Asymptotically stable steady states. HHH Unstable steady states. 00 Orbitally asymptotically stable periodic orbits. 00000 Unstable periodic orbits.

far away from the steady state), and the transition through the instability limit is (respectively, is not) smooth, as far as the dynamic response of the catalyst is concerned; therefore, such a response depends qualitatively on the shape of the actual response curve, which is as one of those sketched in Fig. 11 if it exhibits Hopf bifurcation phenomena.

The analysis of the degenerate bifurcations that take place on the lines and vertices of the diagrams of Fig. 10 is beyond the scope of this paper. Such an analysis would provide predictions about the global shape of the bifurcated branches of periodic orbits. The following degeneracies are present in the diagrams of Fig. 10:

(a) On the common boundary of the regions VI and Va, and of the regions $I$ and IIa, two Hopf bifurcation points coalesce; since both bifurcations are supercritical near this line (see sketches IIa and Va of Fig. 11), both branches are connected near the transition, and coalesce as the line is approached from above.

(b) On the boundary of region IV (=IVauIVb), a Hopf bifurcation point approaches a saddle-node point, and the linearized problem has a double eigenvalue. 
The (two-dimensional) universal unfolding of this (codimension two) degeneracy contains Hopf bifurcations and saddle loops as mechanisms leading to periodic orbits (see [14]); therefore, saddle loops are expected to occur near this line.

(c) On the upper boundary of regions I and II ( = Ila $\cup$ IIb $\cup I I c \cup 1 I d$ ), a (static) bifurcation takes place, associated with a cusp singularity.

(d) On the points of intersection of the three above-mentioned lines, a degenerate bifurcation of higher codimension takes place.

(e) On the remaining lines of the diagram, a higher-order Hopf bifurcation takes place, whose analysis requires us to calculate two more terms in the expansions (5.1)-(5.3) and provides the transition from subcritical to supercritical Hopf bifurcation (see [13]).

6. The case $\sigma=O(1)$. In the limit

$$
L \rightarrow \infty, \quad \gamma \beta \rightarrow 0, \quad \nu \rightarrow 0, \quad \sigma=O(1)
$$

two isothermal models are obtained from (1.1), (1.2) by means of an analysis which is similar to that in $\S 2$.

If $\phi^{2} \exp \left(\gamma-\gamma / v_{s}\right)=O(1)\left(v_{s}\right.$ is any steady state temperature of $\left.(1.1),(1.2)\right)$, then one obtains the model (1.1), (1.4). A linear stability analysis of this model leads to qualitatively similar results to those in $\$ 3$.

If $\phi^{2} \exp \left(\gamma-\gamma / v_{s}\right)$ is large (i.e., $\phi^{2} \gg 1$ if $\gamma=O(1)$ ), then the following model is obtained

$$
\begin{aligned}
& \frac{\partial w}{\partial T}=\frac{\partial^{2} w}{\partial \zeta^{2}}-w \exp (\gamma-\gamma / v) \quad \text { in }-\infty<\zeta<0, \\
& w=0 \quad \text { at } \zeta=-\infty, \quad \frac{\partial w}{\partial \zeta}=1 \quad \text { at } \zeta=0, \\
& \frac{d v}{d T}=\Lambda m(1-v)+\Lambda \exp (\gamma-\gamma / v) \int_{-\infty}^{0} w d \zeta,
\end{aligned}
$$

with

$$
\Lambda=\beta \sigma L S_{\Omega} / \phi^{2} V_{\Omega}, \quad m=\nu / \beta \sigma, \quad T=\phi^{2} t, \quad \zeta=\phi \eta .
$$

Here, $\eta$ is a coordinate along the outward unit normal to $\partial \Omega$, and $w$ is the mean value of $\phi u / \sigma$ at time $T$ over the surface $\zeta=$ constant. Observe that (6.1)-(6.3) differ from the model (1.6) $\sim(1.8)$ only in the boundary condition at $\zeta=0$. The new boundary condition makes it possible to reduce the analysis of the global dynamic behavior of (6.1) $\sim(6.3)$ to the following two-dimensional dynamical system:

$$
\begin{aligned}
& \frac{d W}{d T}=1-W \exp (\gamma-\gamma / v), \\
& \frac{d v}{d T}=\Lambda m(1-v)+\Lambda W \exp (\gamma-\gamma / v),
\end{aligned}
$$

where $W=\int_{-\infty}^{0} w d \zeta((6.4)$ is obtained upon integration of (6.1) and substitution of the boundary condition (6.2)).

The model (6.4), (6.5) may be obtained also from the equations of a continuous stirred tank reactor without cooling (see, e.g., [6]) in the limit of large Damköhler and Lewis numbers. Also, model (6.1)-(6.3) (and, hence, model (6.4), (6.5)) may be obtained from (1.6)-(1.8) in the limit $l \rightarrow \infty, \Phi_{s}^{2}=\Phi^{2} \exp \left(\gamma-\gamma / v_{s}\right) \rightarrow \infty\left(\Phi^{2} \rightarrow \infty\right.$ if 
$y=O(1))$; a simple singular perturbation analysis shows that, after an initial time stage, $U(\xi, \tau)$ and $v(\tau)$ are given by $(6.1)-(6.3)$, with

$$
\Lambda=l / \Phi^{2}, \quad \zeta=\Phi \xi, \quad T=\Phi^{2} \tau, \quad w=\Phi U .
$$

Model (6.4), (6.5) has a unique steady state,

$$
W=\exp [-\gamma /(m+1)], \quad v=1+1 / m,
$$

that is tinearly asymptotically stable if $\gamma \leqq(m+1)^{2} / m$ or if $\gamma>(m+1)^{2} / m$ and

$$
\Lambda m\left[\gamma m /(m+1)^{2}-1\right]<\exp [\gamma /(m+1)],
$$

while it is unstable otherwise. At neutral stability points, there is a Hopf bifurcation, which is subcritical if $m<9-2 \sqrt{6}=0.515$ and $\max \left\{\gamma, \gamma^{*}\right\}<\gamma<\gamma_{+}$, where

$$
\gamma_{1}=(m+1)\left[m+3 \pm \sqrt{m^{2}-18 m+9}\right] / 2 m, \quad \gamma^{*}=(m+1)^{2} / m
$$

while it is supercritical if $\frac{1}{2}<m \leqq 9-2 \sqrt{6}$ and $\gamma^{*}<\gamma<\gamma$, or if $0<m \leqq 9-2 \sqrt{6}$ and $\gamma>\gamma_{+}$, or if $m>9-2 \sqrt{6}$ and $\gamma>\gamma^{*}$, as is easily seen. These results do match with those obtained in $\$ 6$ for $\Phi_{s}^{2}=\Phi^{2} \exp \left(\gamma-\gamma / v_{s}\right) \sim l \rightarrow \infty$.

It is easily seen also that as $\Lambda \rightarrow \infty$, the system (6.4), (6.5) exhibits relaxation oscillations provided that $\gamma>\gamma^{*}$, (i.e., provided that its unique steady state is unstable as $\Lambda \rightarrow \infty$ ).

7. Concluding remarks. Two isothermal models have been derived from the nonisothermal model (1.1), (1.2). They are appropriate for the analysis of nonlinear stability of the steady states of (1.1), (1.2) under small perturbations in the limit $\gamma \beta \rightarrow 0, \nu \rightarrow 0$, $L \rightarrow \infty, \sigma \rightarrow \infty$. If, in addition, $\gamma=O(1)$, then they are also valid in studying global stability properties.

The linearized stability of the steady states for Models 1 and 2, and local Hopf bifurcation for Model 2, have been considered in $\$ \S 3-5$. Some remarks about the results are in order:

(a) For a fixed value of $\mu$, the linear stability diagram of Model 1 for the slab geometry is as one of those in Fig. 2. Oscillatory instabilities appear in a region of the response curve if and only if $\lambda_{\mu}>8.889 \cdots$ and $\gamma_{c 1}<\gamma<\gamma_{c 2}$, where $\gamma_{c 1}=$ $\min \left\{\gamma_{1}, \gamma_{2}, \gamma_{3}\right\}$ and $\gamma_{c^{2}}=\max \left\{\gamma_{1}, \gamma_{2}\right\}$.

(b) For arbitrary shapes of the catalyst in two and three dimensions, it has been shown in $\$ 3.2$ that oscillatory instabilities do not appear if $\lambda \mu$ is sufficiently small or if $\gamma$ is sufficiently small or large (for fixed values of $\lambda$ and $\mu$ ), while they do appear in a region of the response curve if $\gamma>4$ and $\lambda$ is sufficiently large (for fixed values of $\gamma$ and $\mu$ ). This result makes it reasonable to conjecture that for arbitrary shapes of the catalyst, linear stability diagrams are qualitatively similar to those obtained in $\$ 3.1$ for the slab geometry.

(c) For a fixed value of $m$, the linear stability diagram of Model 2 is as that in Fig. 6, which is plotted in Figs. 8 and 9 in the limits $m \rightarrow 0$ and $m \rightarrow \infty$, respectively. Oscillatory instabilities appear in a region of the response curve if and only if $\gamma>\gamma_{c}=$ $\min \left\{\gamma_{1}, \gamma_{2}\right\}$.

(d) It has been shown for lumped chemically reacting systems and conjectured for distributed systems (Ray and Hastings [6]) that a given steady state of the lower or upper segments of the response curve is stable or unstable according to whether the Lewis number is smaller or larger than some bifurcation value (that can be infinite). This conjecture is true for Model 1 in the slab geometry and for Model 2. In fact, for Model 1 for example, the instability bounds, $\phi_{s \mathrm{H} 1}$ and $\phi_{s \mathrm{H} 2}$, are such that $\phi_{\mathrm{sH}}$ decreases and $\phi_{s \mathrm{H} 2}$ increases as $\lambda$ increases (for fixed values of $\gamma$ and $\mu$ ), as seen from the monotonous dependence on $\lambda$ of the lower curve of Fig. 1. 
(e) In the limit $l \rightarrow \infty, m=O(1)$, it is easily seen that the lower curve of Fig. $s$ has two distinguished parts. For $\Phi, \sim 1$, it is given by

$$
\gamma=\left[m+(m+1) \Phi_{s}\right]^{2} / m \Phi_{s}\left(1+\Phi_{s}\right)
$$

in first approximation, while for $1 \ll \Phi_{s}<\Phi_{s}=2 m l+O(1)$, it is given by

$$
\gamma=(m+1)^{2} / m+(m+1)^{2} \Phi_{5}^{2} / m^{2} l .
$$

Hence, if $m<1$ and $4<\gamma<(m+1)^{2} / m$, then

$$
\Phi_{\mathrm{sH} 2} \rightarrow[\gamma-2(m+1)+\sqrt{\gamma(\gamma-4)}] / 2\left[(m+1)^{2} / m-\gamma\right] \text { as } l \rightarrow \infty \text {, }
$$

while $\Phi_{s \mathrm{H} 2} \rightarrow \infty$ as $l \rightarrow \infty$ if $\gamma \geqq(m+1)^{2} / m$ (if $m \geqq 1$ and $4<\gamma<(m+1)^{2} / m$, then every steady state is linearly stable and the point $\mathrm{H} 2$ does not exist). Therefore, there exists a critical value of the activation energy, $\gamma^{*}=(m+1)^{2} / m$, such that if $\gamma \geqq \gamma^{*}$ then the upper instability bound, $\phi_{\mathrm{H} 2}$, is such that $\phi_{\mathrm{H} 2} \rightarrow \infty$ as $l \rightarrow \infty$ (for fixed values of $\gamma$ and $m)$. The same type of behavior is found for the isothermal model $(1.1),(1.4)$ in arbitrary domains, for finite values of $\sigma$ and for more general kinetic laws (see [4]).

(f) In the limit $\Phi \rightarrow \infty$, Model 2 is reduced globally to a two-dimensional dynamical system that exhibits relaxation oscillations as $l / \Phi^{2} \rightarrow \infty$ if $\gamma>\gamma^{*}=(m+1)^{2} / m$, as was shown in $\$ 6$. We may ask whether Models 1 and 2: (i) possess a two-dimensional global attractor, and (ii) exhibit relaxation oscillations as $\lambda \rightarrow \infty$ or $l \rightarrow \infty$ whenever every steady state becomes unstable in that limit. Although it may be proved that Models 1 and 2 possess a finite-dimensional global attractor by using results for more general reaction-diffusion problems, finding precise bounds on the dimension of the attractor is not an easy task (see [15] and references given therein). It seems that the answer to the second question requires an involved multiple time scales analysis that would be much facilitated if an answer to the first question were available.

The above-mentioned dynamical system is obtained also for $\sigma=O(1)$ if $\phi^{2}$ is sufficiently large, as was seen in $\$ 6$. This system is a submodel of the widely uised model of continuous stirred tank reactor without cooling, but differs quantitatively from the system that one would obtain by means of lumping procedures (see, e.g., [1]), as could be expected.

Appendix A. Let $\psi$ be an analytic function inside and on a closed contour $\Gamma$ of the complex plane, without zeros on $\Gamma$. The argument principle (see e.g. Ahlfors [16]) provides the number of zeros of $\psi$ inside $\Gamma$, as $N=\Delta_{\mathrm{I}}$ arg $\psi / 2 \pi$, where $\Delta_{\mathrm{I}}$ arg $\psi$ is the change in value of the argument of $\psi$ when $\Gamma$ is transversed once in the positive sense. In order to calculate the number of roots of (3.1) in the right-hand side of the complex plane, we write (3.1) in the form

$$
\psi(\omega) \equiv \omega^{2}+H \omega+K\left[\left(\tanh \phi_{s}\right) / \phi_{s}-\left(\tanh \sqrt{\omega+\phi_{s}^{2}}\right) / \sqrt{\omega+\phi_{s}^{2}}\right]=0
$$

where

$$
H=\lambda \mu-K\left(\tanh \phi_{s}\right) / \phi_{s}^{3}, \quad K=2 \lambda \mu^{2} \gamma \phi_{s}^{4} /\left(\mu+2 \phi_{s} \tanh \phi_{s}\right)^{2},
$$

and consider a contour $\Gamma$ consisting of four segments: $\Gamma_{1}: \omega=R \exp (i \theta)$, for $-\pi / 2<$ $\theta<\pi / 2 ; \Gamma_{2}: \omega=i \Omega$, for $R \geqq \Omega \geqq r ; \Gamma_{3}: \omega=r \exp (i \theta)$, for $\pi / 2>\theta>-\pi / 2$; and $\Gamma_{4}: \omega=$ $i \Omega$ for $-r \geqq \Omega \geqq-R$. Any root of $\psi$ such that $\operatorname{Re} \omega>0$ is inside $\Gamma$ if $r$ is sufficiently small and $R$ is sufficiently large.

From the asymptotic behavior of $\psi$ as $|\omega| \rightarrow 0$ and as $|\omega| \rightarrow \infty$, it is easily seen that

$$
\Delta_{1,} \arg \psi=2 \pi+\varepsilon_{1}, \quad \Delta_{r_{1}} \arg \psi=-\pi+\varepsilon_{3},
$$

where $\varepsilon_{1} \rightarrow 0$ as $R \rightarrow \infty$ and $\varepsilon_{3} \rightarrow 0$ as $r \rightarrow 0$. 
In order to calculate $\Delta$ arg $\psi$ on $\Gamma_{2}$ and $\Gamma_{4}$, let us consider the functions $\Omega \rightarrow J_{1}(\Omega) \equiv$ $\operatorname{Re} \psi(i \Omega)$ and $\Omega \rightarrow J_{2}(\Omega)=\operatorname{Im} \psi(i \Omega)$, which are given by

(A.4) $J_{1}(\Omega)=-\Omega^{2}+K\left[\left(\tanh \phi_{s}\right) / \phi_{s}-2(a \sinh a+b \sin b) /\left(a^{2}+b^{2}\right)(\cosh a+\cos b)\right]$,

(A.5) $J_{2}(\Omega)=H \Omega+2 K(b \sinh a-a \sin b) /\left(a^{2}+b^{2}\right)(\cosh a+\cos b)$, where $a+i b=2 \sqrt{\phi_{s}^{2}+i \Omega}$, as in $\$ 3.1$.

Since $J_{1}$ is an even function of $\Omega$, and $J_{2}$ is an odd function of $\Omega$, it is clear that

$$
\Delta_{\mathrm{r}_{2}} \text { arg } \psi=\Delta_{\mathrm{r}_{4}} \text { arg } \psi \text {. }
$$

On the other hand, for a fixed value of $\phi_{s}$, the equations $J_{1}=0$ and $J_{2}=0$ define two functions,

$$
K=f_{1}(\Omega), \quad H / K=f_{2}(\Omega),
$$

respectively, which are easily calculated from (A.4), (A.5), and are seen to be monotonously increasing. Furthermore, $f_{1}(0)=K_{m}, f_{2}(0)=H_{m} / K_{m}$, where

$$
K_{m}=\frac{16 \phi_{s}^{5} \cosh ^{2} \phi_{s}}{3 \sinh 2 \phi_{s}-6 \phi_{s}-4 \phi_{s}^{2} \tanh \phi_{s}}>0, \quad \frac{H_{m}}{K_{m}}=\frac{2 \phi_{s}-\sinh 2 \phi_{s}}{4 \phi_{s}^{3} \cosh ^{2} \phi_{s}}<0,
$$

and $f_{1} \rightarrow \infty, f_{2} \rightarrow 0$ as $\Omega \rightarrow \infty$. Therefore, the curve $H=f_{1}(\Omega) f_{2}(\Omega), K=f_{1}(\Omega), 0<\Omega<\infty$, is as the upper curve of the sketch of Fig. 12, and consists of points $(H, K)$ for which (A.1) has a pair of purely imaginary roots, $\omega= \pm i \Omega$. For points of the lower curve of Fig. 12, which is the straight line $K / H=K_{m} / H_{m}$, (A.1) has $\omega=0$ as a double root. Now, $\Delta$ arg $\psi$ along $\Gamma_{2}$ is easily calculated, for $(H, K)$ in regions $A, B$, and $C$ of Fig. 12 , from the shape of the graph of the function $\Omega \rightarrow F(\Omega) \equiv \operatorname{Im} \psi(i \Omega) / \operatorname{Re} \psi(i \Omega) \equiv$ $J_{2}(\Omega) / J_{1}(\Omega)$, for $0<\Omega<\infty$.

If $(H, K)$ belongs to region $\mathrm{A}$, two cases must be considered. If $K \leqq K_{m}$, then $F>0$ for $0<\Omega<\infty, F \rightarrow+\infty$ as $\Omega \rightarrow 0$ and $F \rightarrow 0$ as $\Omega \rightarrow \infty$. If $K>K_{m}$, then $J_{1}\left(\Omega_{1}\right)=0$ for a certain $\Omega_{1}$ such that $0<\Omega_{1}<\infty, F<0$ for $0<\Omega<\Omega_{1}, F>0$ for $\Omega_{3}<\Omega<\infty$, $F \rightarrow-\infty$ as $\Omega \rightarrow 0$ and as $\Omega \rightarrow \Omega_{1}^{-}, F \rightarrow+\infty$ as $\Omega \rightarrow \Omega_{1}^{+}$and $F \rightarrow 0$ as $\Omega \rightarrow \infty$. Therefore, in both cases

$$
\Delta_{\mathrm{Y}_{2}} \arg \psi=\pi / 2+\varepsilon_{2} \quad \text { for }(H, K) \in A,
$$

where $\varepsilon_{2} \rightarrow 0$ as $R \rightarrow \infty$ and $r \rightarrow 0$.

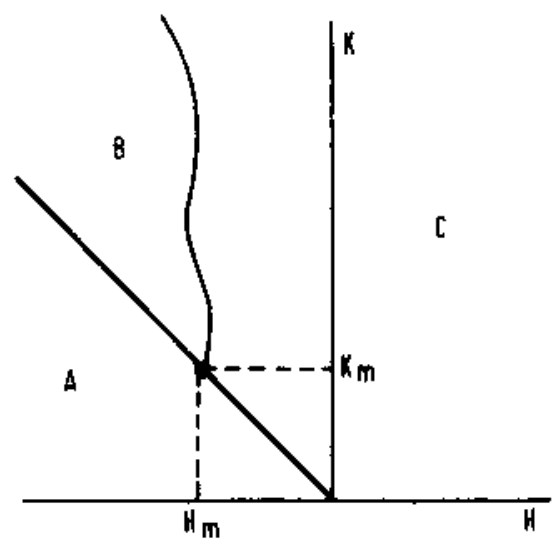

FIG. 12. Roots of (3.1) in the right-hand side of the complex plane. 
If $(H, K)$ belongs to region $B$, then $F\left(\Omega_{1}\right)=J_{2}\left(\Omega_{1}\right)=J_{1}\left(\Omega_{2}\right)=0$ for certain $\Omega_{1}$ and $\Omega_{2}$ such that $0<\Omega_{1}<\Omega_{2}<\infty, F>0$ for $0<\Omega<\Omega_{1}$ and for $\Omega>\Omega_{2}, F<0$ for $\Omega_{1}<\Omega<$ $\Omega_{2}, F \rightarrow+\infty$ as $\Omega \rightarrow 0$ and as $\Omega \rightarrow \Omega_{2}^{+}, F \rightarrow-\infty$ as $\Omega \rightarrow \Omega_{2}^{-}$and $F \rightarrow 0$ as $\Omega \rightarrow \infty$. Therefore

$$
\Delta_{\mathrm{r}_{2}} \arg \psi=3 \pi / 2+\varepsilon_{2} \quad \text { for }(H, K) \in B \text {, }
$$

where $\varepsilon_{2} \rightarrow 0$ as $R \rightarrow \infty$ and $r \rightarrow 0$.

If $(H, K)$ belongs to region $C$, two cases must be considered. If $K \leqq K_{m}$, then $F \rightarrow-\infty$ as $\Omega \rightarrow 0, F \rightarrow 0$ as $\Omega \rightarrow \infty$, and either $F<0$ for $0<\Omega<\infty$ (if $H \geqq 0$ ) or $F<0$ for $0<\Omega<\Omega_{1}$ and $F>0$ for $\Omega>\Omega_{1}$, for a certain $\Omega_{1}$ such that $0<\Omega_{1}<\infty$ (if $H<0$ ). If $K>K_{m}$, then $J_{1}\left(\Omega_{1}\right)=0$ for a certain $\Omega_{1}$ such that $0<\Omega_{1}<\infty, F \rightarrow+\infty$ as $\Omega \rightarrow 0$ and as $\Omega \rightarrow \Omega_{1}^{-}, F \rightarrow-\infty$ as $\Omega \rightarrow \Omega_{1}^{+}, F \rightarrow 0$ as $\Omega \rightarrow \infty, F>0$ for $0<\Omega<\Omega_{1}$ and either $F<0$ for $\Omega_{1}<\Omega<\infty$ (if $H \geqq 0$ ) or $F<0$ for $\Omega_{1}<\Omega<\Omega_{2}$ and $F>0$ for $\Omega>\Omega_{2}$, for a certain $\Omega_{2}$ such that $\Omega_{1}<\Omega_{2}<\infty$ (if $H<0$ ). Therefore, in both cases

$$
\Delta_{\Gamma_{2}} \arg \psi=-\pi / 2+\varepsilon_{2} \quad \text { for }(H, K) \in C,
$$

where, again, $\varepsilon_{2} \rightarrow 0$ as $R \rightarrow \infty$ and $r \rightarrow 0$.

Now, from (A.3), (A.6)-(A.9), we obtain

$$
\Delta_{\mathrm{T}} \arg \psi=2 \pi+\varepsilon, \quad \Delta_{\mathrm{T}} \arg \psi=4 \pi+\varepsilon, \quad \Delta_{\Gamma} \arg \psi=0+\varepsilon,
$$

for $(H, K)$ in regions $\mathrm{A}, \mathrm{B}$, and $\mathrm{C}$ respectively, where $\varepsilon \rightarrow 0$ as $R \rightarrow \infty$ and $r \rightarrow 0$. Then, by taking $R^{-1}$ and $r$ sufficiently small, it turns out that the number of zeros of (A.1) in the right-hand side of the complex plane is one, two, and zero, for $(H, K)$ in regions $\mathrm{A}, \mathrm{B}$, and $\mathrm{C}$ of Fig. 12, respectively, or for $\left(\phi_{s}, \gamma\right)$ in regions $\mathrm{A}, \mathrm{B}$, and $\mathrm{C}$ of Fig. 1, respectively, which are mapped in their homonyms of Fig. 12, through the functions (A.2).

A completely similar analysis applies to (4.1) of $\$ 4$.

Appendix B. In this Appendix we derive some technical results which are used in \$3.2. Maximum principles (Protter and Weinberger, [17]) will be widely used in the sequel.

Inequalities (3.10) and (3.16) are obtained by standard arguments, using maximum principles. In order to obtain (3.11) and (3.17), let $q$ be any point of $2 \Omega$, let $S_{1}$ and $S_{2}$ be the hyperspheres, of radius $\rho_{1}$ and $\rho_{2}$, to which the interior and exterior sphere properties refer, and let $x_{2}$ be the center of $S_{2}$. Standard maximum principles applied to (3.7) show that

$$
u_{s}(x)>\exp \left[\delta\left(\rho_{2}-r\right)\right] \text { for all } x \in \Omega,
$$

where $r=\overline{x_{2} x}$ and $\delta=\phi_{s}+(p-1) / \rho_{2}$. From (B.1), inequality (3.11) is readily obtained; in addition, $u_{s}$ satisfies: $u_{s}(x)>\exp (-1)$ if $r-\rho_{2}<1 / \delta$. Then, if $S_{0}$ is the hypersphere, of radius

$$
\rho_{0}=\min \left\{\rho_{1}, 1 / 2 \delta\right\}=\min \left\{\rho_{1}, \rho_{2} / 2\left(p-1+\rho_{2} \phi_{s}\right)\right\},
$$

which is tangent to $\partial \Omega$ at $q$ and satisfies $S_{0} \subset \Omega$, we have: $u_{s}(x)>1 / e$ for all $x \in S_{0}$. Therefore, if $x_{0}$ is the center of $S_{0}$ and if $r=\overline{x_{0} x}$, standard maximum principles applied to the solution of (3.15) in $S_{0}$ show that

$$
\bar{u}(x)<\left(2 / e \phi_{s}\right)\left\{\exp \left[\delta_{0}\left(r^{2}-\rho_{0}^{2}\right)\right]-1\right\} \text { for all } x \in S_{0},
$$

where $\delta_{0}=\phi_{s}^{2} / 2\left(p+\rho_{0} \phi_{s}\right)$. Hence

$$
\left(\frac{\partial \bar{u}}{\partial n}\right)_{q}>2 \rho_{0} \phi_{s} / e\left(p+\rho_{0} \phi_{s}\right) .
$$

From (B.2) and (B.3), inequality (3.17) readily follows. 
In order to prove property (iv), let $\omega$ be an eigenvalue of $(3.20),(3.21)$, and let $Z$ be the corresponding eigenfunction. If $b=\operatorname{Im} \omega \neq 0$, then $a=\operatorname{Re} \omega, Z_{R}=\operatorname{Re} Z$ and $Z_{i}=\operatorname{Im} Z / b$ satisfy

$$
\Delta Z_{R}-\left(a+\phi_{s}^{2}\right) Z_{R}+b^{2} Z_{I}=\gamma v_{\mathrm{s}}^{2} \phi_{\mathrm{s}}^{2} u_{\mathrm{s}} \quad \text { in } \Omega, \quad Z_{R}=0 \quad \text { on } \partial \Omega,
$$

$$
\Delta Z_{f}-\left(a+\phi_{s}^{2}\right) Z_{I}-Z_{R}=0 \text { in } \Omega, \quad Z_{I}=0 \quad \text { on } \partial \Omega \text {, }
$$

$$
a / \lambda+\mu=\phi_{s}^{2} \int_{\Omega} Z_{R} d x+\gamma \mu\left(v_{s}-1\right) / v_{x}^{2}, \quad 1 / \lambda=\phi_{s}^{2} \int_{\Omega} Z_{1} d x .
$$

If $\omega$ is a real double eigenvalue of (3.20), (3.21) and $Z$ is the corresponding (real) eigenfunction, then (B.4)-(B.6) hold with $Z_{R}=Z, Z_{i}=\partial Z / \partial \omega, a=\omega$, and $b=0$. Therefore, to prove property (iv), we only need to show that inequality (3.22) holds if (B.4)-(B.6) have a solution for some $a \geqq 0$ and $b \geqq 0$.

Integration over $\Omega$ of the product of $Z_{l}$ by (B.5) yields

$$
\int_{\Omega}\left|\nabla Z_{j}\right|^{2} d x+\left(a+\phi_{s}^{2}\right) \int_{\Omega} Z_{j}^{2} d x=-\int_{\Omega} Z_{R} Z_{l} d x
$$

where $\nabla$ is the gradient operator. Similarly, integration over $\Omega$ of the equation, which is obtained when (B.4) is multiplied by $-\left(Z_{R}+a Z_{1}\right)$, (B.5) is multiplied by $\left[a Z_{R}-b^{2} Z_{l}+\gamma v_{s}^{-2} \phi_{s}^{2}\left(u_{s}-1\right)\right],(3.7)$ is multiplied by $-\gamma v_{v}^{2} \phi_{r}^{2} Z_{I}$, and the resulting equations are added, leads to

$$
\gamma v_{s}^{-2} \phi_{s}^{2}\left[\int_{\Omega} Z_{R} d x+\left(a+\phi_{s}^{2}\right) \int_{\Omega} Z_{I} d x\right]+\int_{\Omega}\left|\nabla Z_{R}\right|^{2} d x+\phi_{s}^{2} \int_{\Omega} Z_{R}^{2} d x \leqq 0 .
$$

On the other hand, Poincaré inequality (see, e.g., Gilbarg and Trudinger [18]) and Schwarz inequality yield

$$
\begin{aligned}
& \text { (B.9) } k \int_{\Omega} Z_{R}^{2} d x \leqq \int_{\Omega}\left|\nabla Z_{R}\right|^{2} d x, \quad k \int_{\Omega} Z_{l}^{2} d x \leqq \int_{\Omega}\left|\nabla Z_{l}\right|^{2} d x \\
& \text { (B.10) }\left|\int_{\Omega} Z_{R} Z_{I} d x\right| \leqq\left[\int_{\Omega} Z_{R}^{2} d x \int_{\Omega} Z_{l}^{2} d x\right]^{1 / 2}, \quad\left|\int_{\Omega} Z_{I} d x\right| \leqq\left[V_{\Omega} \int_{\Omega} Z_{I}^{2} d x\right]^{1 / 2},
\end{aligned}
$$

where $k$ is given by (3.23). Substitution of (B.9), (B.10) into the second equation (B.6) and into (B.7), and multiplication of the resulting equations lead to

$$
\int_{\Omega} Z_{k}^{2} d x \geqq\left(\phi_{s}^{2}+k\right)^{2} / \lambda^{2} \phi_{s}^{4} V_{\Omega} .
$$

Finally, substitution of (B.6), (B.9), (B.11) into (B.8) yields the inequality (3.22).

The inequalities (3.24) are easily obtained when maximum principles are applied to $(3.20)$. Property (3.25) comes out from the inequalities

$$
0 \leqq-\left(\omega+\phi_{s}^{2}\right) \int_{\Omega} Z(\omega, x) d x \leqq \gamma v_{\mathrm{s}}^{-2} \phi_{s}^{2} \int_{\Omega} u_{s} d x,
$$

which are readily obtained, upon integration of (3.20), when taking into account that $\partial Z / \partial n \geqq 0$ for all $x \in \partial \Omega$ and all $\omega \geqq 0$, as it comes out from (3.24).

\section{REFERENCES}

[1] R. ARIS, The Mathematical Theory of Diffusion and Reaction in Permeable Catalysts, Vol. I and Il, Clarendon Press, Oxford, 1975. 
[2] N. R. Amundson AND L. R. RAYMOND, Stability in distributed parameter systems, Amer. Inst. Chem. Engrg. J., 11 (1965), pp. 339-350.

[3] P. H. Nielsen AND J. VILLADSEN, Absorption with exothermic reaction in a falling film column, Chem. Engrg. Sci., 38 (1983), pp. 1439-1454.

[4] J. M. VEGA, Invariant regions and global asymptotic stability in an isothermal catalyst, SIAM J. Math. Anal., 19 (1988), pp. 774-796.

[5] S. P. HASTINGS, Formal relaxation oscillations for a model of a catalytic particle, Quart. Appl. Math., 41 (1984), pp. 395-405.

[6] W. H. RAY AND S. P. HASTINGS, The influence of the Lewis number on the dynamics of chemically reacting systems, Chem. Engrg. Sci, 35 (1980), pp. 589-595.

[7] D. S. COHEN AND A. B. POORE, Tubular chemical reactors: the lumping approximation and bifurcation of oscillatory states, SIAM J. Appl. Math., 27 (1974), pp. 416-429.

[8] J. D. MUrRaY, A simple method for obtaining approximate solutions for a class of diffusion-kinetic enzyme problems: II. Further examples and nonsymmetric problems, Math. Biosci., 3 (1968), pp. 11S-133.

[9] 5. E. Marsden and M. MCCracken, The Hopf Bifurcation and its Applications, Springer-Verlag, Berlin, New York, Heidelberg, 1976.

[10] B. D. Hassard, N. D. Kazarinoff and Y. H. WaN, Theory and Applications of Hopf Bifurcation, Cambridge University Press, London, Cambridge, 1981.

[11] D. HENRY, Geomesric Theory of Semilinear Parabolic Equations, Springer-Verlag, Berlin, New York, Heidelberg, 1981.

[12] H. KIElHöfER, Generalized Hopf bifurcation in Hibert space, Math. Methods Appl. Sci., 1 (1979), pp. 498-513.

[13] M. GOLUBITSKY AND W. F. LANGFORD, Classification and unfoldings of degenerate Hopf bifurcations, J. Differential Equations, 41 (1981), pp. 375-415.

[14] J. GuCK FNhEIMER, Multiple bifurcation problems of codimension fwo, SIAM J. Math. Anal., 15 (1984), pp. 1-49.

[15] N. KOPELL AND D. RUELLE, Bounds on complexity in reaction-diffusion sustems, SlAM J. Appl. Math., 46 (1986), pp. $68-80$.

[16] L. V. Ahlfors, Complex Analysis, McGraw-Hill, New York, Toronto, London, 1966.

[17] M. Protter and H. Weinberger, Maximum Principles in Differential Equations, Prentice-Hall, Englewood Cliffs, NJ, 1967.

[18] D. Gilbarg and N. S. TRudinger, Elliptic Partial Differential Equations of Second Order, SpringerVerlag, Berlin, New York, Heidelberg, 1977. 